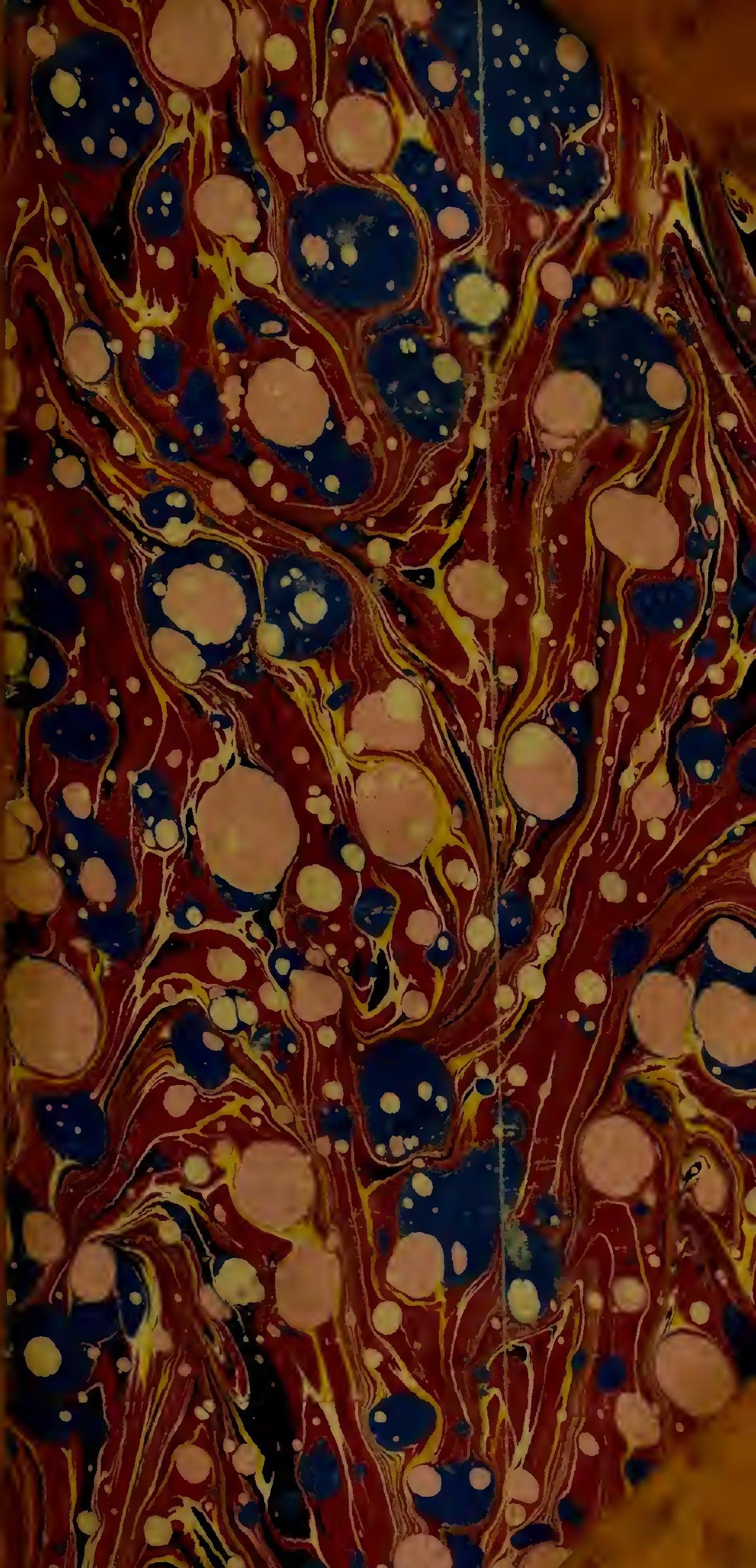


08.9

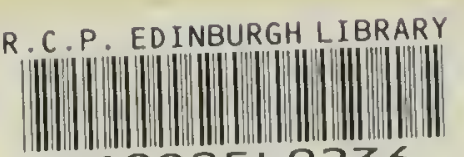



Digitized by the Internet Archive in 2015 




\section{DE LA DISTINCTION ORIGINELLE}

DU

\section{TESTIGULE ET DE L'OVAIRE;}

CARACTÉRE SEXUEl DES DEUX FEULLETS PRIMORHALX DE L'EMBRYON; IIERMAPIIRODISME MORPIOLOGIQUE DE TOUTE INDIVIDCALITÉ ANIMALE; ESSAI D'UNE TULORIE DE L.I FÉCONDATIOX;

IAl

\section{ÉdDUARD VAN BENEDEN,}

professeur a L'Univensté de liégb, membre de L'académie royal DE BELGIQUE.

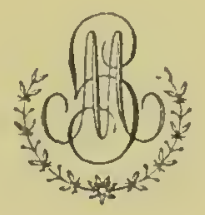

\section{BRUXELLES,}

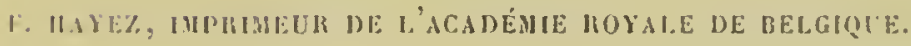

1874. 
Extrait des Bubletins de l'Académie royale de Belgique, gme série, tome XXXVII, no 3 ; mai 187 i. 


\title{
DE LA DISTINCTION ORIGINELLE
}

DU

\section{TESTICULE ET DE LOVAIRE;}

\author{
CARACTĖRE SEXUEL DES DEUX FEULLETS PRHORDALX DE \\ L'EMBRYON; HERMAPURODISME MORPHOLOGIQUE DE TOUTE \\ INDIYIDUALITÉ ANINALE; ESSAI D'UXE THEORIF UE LA \\ FÉCONDATION.
}

\section{PREMIERE PARTIE.}

1. Introduction. Homologie de l'eetoderme et de l'endoderme dans les divisions: primaires du règne animal. II. Historique des recherches faites sur l'origine des produits sexuels chez les Polypes (1). III. Études sur l'Ilydractinir echinata.

\section{I. - Inthoduction.}

Ifuxley fut le premier qui démontra que tout l'organisme des Zoophytes, Méduses et Polypes, Hydrö̈les et Siphonophores peut se ramener à un sac formé de deux conches cellulaires adjacentes: l'ectoderme et l'endoderme (Allman) et qui considéra cette proposition comme exprimant la loi générale de structure chez les Zoophytes (2). Quoique

(1) J'emploie celte dénomination dans le sens que mon père lui a altribué (1840) pour désigner les Polypes, les Acalèphes el les Spongiaires; comme synonyme de Coclenlèés (1 \$ 17 , Leuckarl) el de Zoophyles (Hxchel).

(2) Observations upon the analomy of the Diphyde and the unity of organisation of the Diphydre and Siphonophora (Envoyed'suslnalie? is lit 


\section{(4)}

l'on ne songeât pas encore ì celte époque à rechercher des loomologies entre les Vertébrés et les animaux inférieurs, Husley comprit toute la portée de sa décourerte. Il reconnut et formula dans un langage clair et précis sou opinion surr l'lomologie qu'il crovait exister entre l'ectoderme et l'endoderme des Coclentérés et les deux feuillets cellulaires primordiaux des vertćlrés.

Voici en quels termes il exprina celte idée :

"The peculiarity in the structure of the body-walls of the IIjdrozoa, to which I lave just referred, possesses a singular interest in its bearing upon the truth that there is a certain similarity between the adult states of the lower animals and the embryonic conditions of higher organisation.

For it is well linown that, in a rery early state, the germ, even of the highest animals is a more or less complete sae, whose thin wall is divisible into two membranes, an inner and an outer; the latter, turned towarls the extcrnal world; the former, in relation with the nutritive liquid, the yelli.

The various organs are produced by a process of budding from one, or other, or both of these primary liacers of the germ. 》

Il cherche mème a établir un parallélisme, au point de vue histogénique, entre l'ectoderme des Zoophrytes et li feuillet externe de l'embryon des rertébrés d'un côté,

Limncan Society en 184i el public dans les Procernaxgs or tue lioyu. socretr en juin 1849).

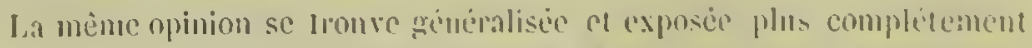
dans nn travail public ha meme annes, Memoir on the anatom! and afinilies of the Medusar. 


\section{(}

l'endoderme et le feuillet interne de l'autre. Il conclut en disant: "Thus there is a very real and genuine analog!, between the adult Hydrozoon and the entbryonic vertebrate animal.

Toutes les recherclıes embrỵgéniques faites dans ces dernicres années sur les premières phases du développement cubryonnaire des animaux de tous les embranchements, sont venues confirmer', en l'étendaut à l'ensemble du rigne animal, l'opinion de l'illustre naturaliste anglais. Et an premicr rang des travaux faits dans cette direction se placent sans contredit ceux de Kowalewshy : en montrant l'identité du développement de l'Amphioxus et des Ascilies, il combla d'un seul coup l’ahione jugé infranchissable qui séparait l'enılonehement des vertébrés de tous les organismes inféricurs. Les importantes publicaltions du mème auteur sur les autres ypes d'organisation, jointes it celles de Gegenbauer, de Hreckel, de Ray Lankester, de Kilcinenberg et de quelpues autres, ont eu pour résultat d'étendre à l'ensemble du rigne animal cette grande conception que toutes les parties des organismes animaux s' forment aux dépens de deux feuillets cellulaires primor'diaux, et partout homologues.

Ces idées viennent d'ètre longuement déreloppées el brillamment défendues dans deux écrits d'mne haute portéc philosophique: Hackicl a exposé, dans sa brochure Die Gastrach-Theorie, die phylogenetische Classification des Thierreiches und die Homologie der Keimblälter, une théoric qu’il avait énoncéc tout d'abord dans sa Monograplie les ćponges calcaires. Des idées analogues, et à plusicurs égards tout à lait identiques, ont été publićes en Angleterre dans les Annals and Magazine of natural History sous le titre: "On the Primitive Cell-layers of the Embryo as. 


\section{( 6 )}

the Basis of Genealogical Classification of Animals „ par mon ami E. Ray Lanliester.

Tous les animaux pluriccllulaires, clicz lesquels le: développement débute par le fractionuement de la cellulereuf, passent dans le cours de leur évolution par une mème for'me embryonnaire, celle d'un sac dont les parois minces sont constituées par deux couclies adjacentes; l'endoderme et l'ectoderme. La première circonscrit immédiatement une cávité qui est le tube digestif primordial; la seconde limnite extérieurement le corps de l'eınbryon; elle seule peut ètre impressionnée par les causes externes. - La cavité digestive communique avec l'extérieur jar un seul orifice qui sert à la fois de bouclie el d'anus. - L'embryon se réduit à une cavité digestive, qui n'est qu'un simple estomac; Hackiel a proposé de donner à cette forme primordiale le nom de Gastrula. - Comme cette forme embryonnaire se rencontre chez les Vertébrés aussi bien que chez les Mollusques, les Arthropodes, les Échinodermes, les Vers et les Polypes, il est clair que l'eetoderne est homologue chez les différents types d'organisation; que l'endoderme a chez tous la même valeur morphologique; que la cavité digestive primordiale des Vertébrés et celle de tous les autres types d'organisation ont la même signilication anatomique. Lexistence de cette forme commune dans le cours de l'évolution de tous les animaux métazoaires, permet de les lamener à une souche commune; il y a conver'gence des grands types d'organisation et non parallélisme, comme on l'avait admis depuis Cuvier et ron Bàcr. Enfin. on peut en induire l'existence, à une époque géologique reculée, d'organismes semblables à la forme Gastrula; ces organismes, probablennent variés de mille manières dans leur forme el dans leurs caractères extérieurs, ont été lit 


\section{( 7 )}

souche commune des Vertébrés, des Arthropodes, des Mollusques, des Échinodermes, des Vers et des Zoopliytes; ils constituaient le groupe très-nombreux des Gastrieades (Hackiel).

Si l'endoderme et l'ectoderme sont homologues che\% tuus les llétazoaires, on est en droit de supposer que ces denx feuillets cellulaires ont aussi chez tous la même valcur listogénique et que les mêmes șrstèmes organiques se développent dans les différents types d'organisation aux dépens des mêmes feuillets primitifs.

Cette induction a été déjà pleinement confirmée en cé qui concerne le système nerveux central qui se développe chez tous les animaux aux dépens de l'ectoderme.

Dès lor's, il est indifférent, si l'on veut comnaître l'origine d'un appareil, de la rechercher dans l'un ou l'autre type d'organisation; les résultats pourront s'étendre à l'ensemble du règne animal et recevoir une portée générale.

Or, de tous les types d'organisation celui qui se prête le mieux ì la rechercbe de cette question capitale de l'origine des systèmes organiques, c'est celui des Polypes, encore appelés Zoophytes ou Coelentérés. Chez eux, en effet, l'ectoderme et l'endoderme persistent avec leurs caractères embryonnaires pendant toute la durée de la vie; tous les organes des Zoophytes ne sont qu'une dépendance de l'un ou l'autre de ces feuillets, quelquefois des deux feuillets réunis.

La forme polype se ranìne avec la plus sgrande facilité a la Gastrula dont toutes les parties se conservent sans subir de grandes modifications pendant tout le cours de l'existence. 


\section{$(8)$}

\section{II. - Historique.}

La question de l'origine des organes sexuels, de l'ovaire et du testicule, de l'weuf et du spermatozoïde est peut-être la plus importante de toutes celles dont la solution peut itre donnće par l'llistogénie. Aussi a-t-elle été l’oljet d’un grand nomlıre d'investigations. Elle a été étudiée clıez les Vertébrés, mais plus encore chez les Zooplıyles. I es solıtions (qu'on én a données, basées en apparence sur les recherclies les plus minutieuses, sont si diverses, el en mème temps affirmées avec tant d'assurance, qu'il n'est pas possible, en se fondant sur les renseignements jubliés jusqu'anjourd'luni, de se faire une comviction personnelle, et de prendre parti pour l'une ou pour l'autre opinion. Tandis que les uns soutiennent de la manière la plus positive que les oufs et les spermatozoïdes se forment aux dépeus de l'endoderme, les autres certilient d'une façon aussi absolute' l'origine ectodermique des produits sexuels.

Tous ccux qui se sont occupés de l'organisation des Zoophytes et qui ont étudié, à quelque point de vue que ce soit, leurs organes sexuels, ont reconnu que les œufset les. spermatozoïdes se développent entre l'ectoder'me et l'endoderme. Rien n'est plus facile que de constater quil en est loien ainsi, au moins en apparence, et les sporosacs aussi bien que les Méluses libres laissent aperceroir les aufs et le testicule entre ces deux membranes. Sur ce point, du reste, toutes les affirmations sont unanimes. Les divergences commencent seulement quand il s'agit de décider quelle est l'origine primitive des produits sexuels.

Lorsque Huxley commença la séric de ses publications 


\section{(9)}

sur la morphlologic des IIydrozoaires, Will at Hilue Elwards avaient déjil établi que chez les Méduses les organes scxuels sont toujours en connexion avee gunclque partie dn systeme gastro-vasculaire; mais ces matmralistes n’avaicut pas cherché à déterminer la nature de celtu connexion. Thans son célèbre mémoire sur l’anatomic et les affinités des lléduses (I), Hnxley décrit l’ovaire el le testicule des Cryptocarpéces, des Plianérocarpées et des lihizostomides comne étant formés à la fois par l'ectoderme et par l'endorlerme; il reconnaît que toujours les oufs et les spermatozö̈des se tronveut en voie de déreloppenent entre les den: membranes, sans les faire dériver' ni de l'une ni de l'antre. Dans ses Oceanic IIydrozoa, il se prononce, quoique avec réserve, en favenr de l'origine ectodernique des produits sexuels : "The reproductive organs consist, troughout the Ilydrozoa, of spermaria and ovaria, portions of the tissue of the wall of the liydrosoma, and I believe, more particularly of the cetoderm, melamorphoset! into spermatuana or oca (2). .)

Köllilier (5), au contraire, affirme positivement que les cufs aussi lien que les spermatozoïles se forment invariablement aux dépens des cellules de l'endoderme. Allman a tonjours défendu la mème thèse. II a déclaré, dans un grand nombre de publications successives, que ses observations ne laissent aucun doute relativement à l'origgine endodermique des produits sexucls; il a formulé son opinion dans sa magnifique Honographie des Hydrö̈des g̣m-

(1) On the Analomy and the Affinities of the Family of the Medustr. Pulosolulcal. Trassactioss, 18.49. Part. I, pazes 420 et suivantes.

(2) The Oceanic IIydrozou, publie par la Ray Sociely, 1838, page 16.

15. Icones llistiologicce, 1866. Part. I1, page 8.9. 


\section{$(10)$}

noblastiques (1), et tout récemment encore il a mainten la même manière de voir quand il a rendı compte des recherches de F. E. Schulze (2) et de Kileinenberg (5).

Allman signale la divergence qui existe entre ses obserrations et les résultats auxquels kileinenherg est arrivé par ses études sur l'Ilydre d'eau donce. "Dans les Irỵdrö̈drs marins, dit Allman, les éléments sexuels se forment aux dépens d'me couclie lıomogène, qui apparait entre l'endoderme el l'ectoderme dn manubriun d'un gonophore, ei il faut bien recomnaitre que jusqu’ ì présent nous n’avons pas de raison pour la faire dériver plutôt de l'me que de l'autre des deux membranes. Les oufs et les spermatozö̈les se différencient anx dépens de celte couche gui seépaissit rapidement. Son accroissement sopère comme si elle subissait constamment de nouvelles alditions du còté de l'endoderme, ce qui me porte à croire qu’elle s"éphissit par transformation progressive du tissu condodermil|ne. Toujours est-il que les éléments reproducteur's se développent lans une direction centrifuge, que les ocufs et les spermatozoüdes les plus arancés dans leur développement se trouvent toujours au contact de l'ectoderme et les moins muirs dans le voisinage de l'condoderme. Ces faits ne lémontrent pas d'une manière absolue l'origine endodermique des produits sexuels; mais elles la rendent extremement probable. $D$ Un autre fait qu'Allman inroque en faveur

(1) A Monograph of the Cymmoblastic or Tubularian IIydroüds, 187., lage 149.

(2) Remarlis on Prof. Schulse"s Mrmoir on Cordylophure lacustris.

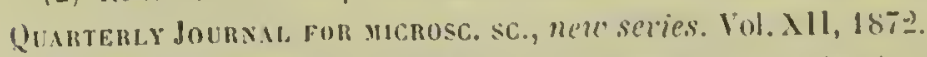

(5) Some alcouml on kleinenhergis liesearches on the Anatomy and itecelopment of IIydra. Imo. 
de son opinion, c'est l'existence, dans certains cas, d'une membrane mince à la face extéricure des produits sexuels. de manière à séparer ceux-ci du contact immédiat de l'ectoderme. Cette membrane, Allman la considere comme n’étant antre chose que la lamelle musculaire de lileinenberg.

Les Spongiaires comme les Coxlentérés sont constitués d’un endoderme et d'un ectoderme. Hiecliel fait dériver de l'endoderme les oufs aussi bien que les spermatozoïdes des Éponges calcaires. Chez les Spongiaires il n'existe ni oraire ni testicule proprement dit; les ouls et les spermatozoüdes penvent se former par différenciation des cellules de l'endoderme en n'importe quel point du système canaliculaire. Cependant Iræckel a conservé un doute relalivement à cette origine, surtout en ce qui concerne les œufs. Les cul's montrent chez tous les Spongiaires des mouvements anœboïdes extrèmement actif's et ils royagent non-seulement à l'intérieur des canaux de l'Éponge, mais mème dans l'épaisseur des tissus; anssi les troure-t-on fréquemment entre l'endoderme et l'ectoderme, voire même au milieu des cellules de cette dernière membrane. Heckel dit explicitement que la question de l'origine et de la situation primordiale des produits sexuels est la plus difficile et lia plus olsscure de toutes celles qui se présentent dans l'étude de l'histologie des Éponges, et il ajoute: a el des Zoophytes en général (1). „

Dès 1864 ses études sur l'organisation et le développements des Geryonides avaient fait dire à Ifreckel que clıe\%

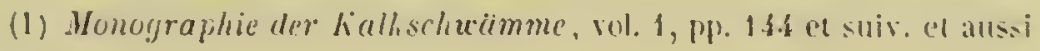
1. 470 . 
res Méduses les wut's aussi bien que les spermatozoïdes se forment aux dépens de l’endoderme (1).

Les recherches de lieferstein et de Elılers (2), de Claus et de P.-E. Müller chez les Siphonophores, de F.-E. Schılze sur tes Cordylophora, enfin de Kleinenberg sur l'Ilydre l'eau donce, tendent à lémontrer, tont an contraire, que les produits sexucls dérivent de l'ectoderme.

lieferstein et Ehlers n'ont pas fait de cette question de l'origine des produits sexnels une étnde spéciale. 1ls affiriment que ces produits naissent du feuillet ectodermique; mais unlle part ils ne rendent compte des olsservations qui lenr ont fait adopter cette manière de voir. ducune description du mode de formation des ouls ou du testicnle n'est dounée dans leur mémoire.

Clans (5) a décrit après IIuxley, Keferstein, Eluters et Gegenbauer te développement d'un bourgeon cellulaire qui, dans les cloches sexueltes des Siphonophores, se forme. anx dépens de lectoderme et qui donnerait naissance. d'après lui, aux produits sexuels màles et femelles.

Tout récemment un excellent observatenr danois, P.-E. Müller', est arrivé à des conchusions inutes sembla-

(1) Hicckel, Die Fumilie der Russelquallen (Medusit Gergunid.e), Jenciische Zeilschrift, I BAI, 186.1, 1. 4.19.

(2) lieferstein ind Bhler's. Zoologische Beilviige : Ier aldsseren Ilaml

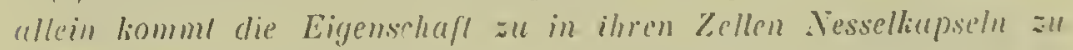
billen, und sis hat mehr de'n Charalite't siner blossen Declie, obwohl auch die Geschlechlsproducte in ils bereiled ueveden, p. a.

(J) Neue Beobachtungen nber die Structur und Entwicliehung der Siphonophoren. Zeilschrift fur weiss. \%ool. Bd. Xll. Il avail deja emis la meme opinion dans son premier mémoire sur l'organisation des siphonopheres: Leber lligsaloptera hydrostatica. Zcilschr. fur wiss. Zool. Bil. I:- 


\section{$(15)$}

lules en étudiant d'autres Siphonophores, principalement l'Ilippopodius luteus (1).

Kileinenberg (2) décrit avec beauconp de délails la for'mation du testicule et de l'ovaire de l'Hydre, aux dépens de son tissu interstiticl qui üest que la partie profonde de l'ectoderme. La formation de l'organe débute par un! accroissement et une prolifération des cellules de ce tissu: l'une de ces cellules s'accroìt exceptionnellement pendant que les autres restent stationnaires; son noỵan se déreloppe et devient la vésicule germinative; son corps se charge d'éléments nutritifs de façon à constituer le vitellus de la cellule-œuf.

Chez le Cordylophora lacustris, F.-E. Scluulze fait apparaitre les oufs dans des épaississements de l'ectoderme. En ce qui concerne les ouf́s, il est très-explicite : il dit mène que dès le début de leur apparilion les œufs se trouvent en dehors de la membrane anlyyste qui sépare l'ectoderme de l'endoderme.

It règne dans les sciences naturelles, et il en est ainsi dans toutes les branches des connaissances humaines, des idées que tout le monde partage, qui sont regardées comme des vérités inébranlables; on les acecpte comme de véritables dogmes sans que l'on sache sur quoi ils reposent ni pourquoi on les admet. Cette croyance dans de prétendus axiomes scientifiques a été cause de bien des crreurs; clle a arrèté hien souvent dans leur marche progressive les sciences d'observation. Au nombre de ces dogmes scientilịnes acceptés comme articles de foi par tous les cmbryo-

(1) Jayllagelser over nogle Siphonophorer. Kjobcnharn, 18 il.

(2) Kleinenberg, IIydra. Eine Anatomisch-entwichelungsgeschichtliche Untersuchung. $18: 2$. 
génistes et tous les physiologistes se trouve la notion de la communauté d'origine de l'ovaire et du testicule. J'étais imbu du mème préjugé quand j’ai comunencé mes recherches, et il a fallu une circonstance particulièrement heureuse pour faire naître le doute dans mon esprit et me permettre de faire un pas vers la comnaissance de la vérité. Quand je me suis rendu à Ostende pour tâcher de me faire une conviction personnelle sur la question de l'origine des produits sexuels clıez les Zoophytes, j’étais loin d'imaginer qu'elle pùt être différente dans les deux sexes. Je fis d'abord toutes mes observations sur des colonies femelles, car les œuffs sont plus faciles à distingner au milieu de n'importe quel tissu que des cellules sperinatiques.

Quand j'eus reconnu de la manière la plus positive chez les Hydractinies, clıez les Clava el chez les Méduses des Campanulaires que les œufs prennent naissance dans l'endoderme, et qu'ils ne sont en dernière analyse que des cellules cndodermiques modifiées, je crus le problème résolı, et j'eus la conviction que les produits sexuels dérivent de l'endoderme. Je me mis alors, à étudier des sporosacs mâles. Je crus reconnaitre que le testicule se forme aux lépens de l'ectoderme. Je ne quittai pas mon microscope avant d'avoir obtenn une certitude complète. Plusieur's fois j’ai refait toutes mes préparations dans les deux genres que jarais sous la main. Il fallut bien se rendre à l'éridence. Alor's je compris la portée de ma découverte. Revenu à Liége, j’ai rérifié encore toutes mes observations sur des Hydractinies que j’avais ramenées virantes.

Si j’ai rappelé assez longuement l'listoire de mes recherches, c'est pour montrer que ce riest pas sous l'influence l’une idée préconçue que j’en suis venu à établir la distinction originelle des deux glandes sexuelles : jétais telle- 


\section{(15)}

ment pénétré de la croyance à la communauté d'origine de l'ovaire et du testicnle, que j’étais au début plus tenté de donter de mes sens que de la vérité du dogme scientifiçue; tant il est difficile de se débarrasser de certaines idées préconçues et tant leur influence est funcste! Et quant anx divergences d'opinions entre les auteurs, je me les explique jusqu’à un certain point par cette considération que la plupart des naturalistes ont fait probablement des recherclies sur l'un des seres et qu'ils ont étendu à l'autre les conclusions de leur étude.

Deux genres d'Hydrö̈des communs sur' nos côtes out fait les principanx frais de mon travail : l'Ilydractinic echinata de P. J. Van Beneden (1) et l'Hydra squamata de 0. F. Mü̈ller (2), la Coryna squamala de Lamarek (5), le type du genre Clava de Gmelin (4), reconnu par Jolnnston (马) et Strethill Wright (6); Hincks (7) a restitué à cet animal son premier nom spécifique: il a désigné ce joli polype sous le nom de Clava squamata qui lui a été conservé dans la monographic des Tuluularides de Allman (8).

(1) P. J. Van Beneden, Bulletin de l'Académie royale de Belyique, t. VIII, $18 \$ 1$.

2o Recherches sur l'embryogenie des Tubulaires, MéMorres DE L'AC.1DEMIE RoYale DE BELGiQte, t. XVIIT, p. 10i, pl. IX.

$\tilde{J}^{\circ}$ Recherches sur la faune lillorale de Be!gique (Polypes). MÉnotres DE L'ACAdéme royate de Belgique, t. XXXVI, p. 151.

(2) Otho Fred. Müller, Zool. Dan. Icon. Tah. IV.

(5) Lamarck. Hisloire des animaux sans verlibres.

(4) Ginelin a créé le genre Clava dans la treizieme édilion du Systema Valure, 1788.

(i) Johnston, British Zooph., 1817, p. 50.

(6) Strethill Wright. Proc. Roy. Phys. Soc. Edimb., rol. I, p. 228, pl. x, figg. 2 et 3.

(7) Ilincks, Bril. IIyllr. Zooph., p. 4, pl. 1, fig. 9?.

(४) Allman. A Monograph of the Gymnoblastic or Tubularian $\mathrm{Hy}$ droids, part. 11. 


\section{III. - Rechemches ser l'IIydractixia ecmixata.}

Les Hydractinies virent en nombreuses colonies it la surface de différentes espèces de copuilles de Gastéropodes (Buccinum, Nalica, et d'autres) abbandonnérs par leurs habitants naturels et occupées par des Pagures. Les pècheurs de crevettes en prennent régulièrement dans leur's filets; el durant tout le temps que j’ai passé à Ostende j’ai pu m'en procurer journellement. On peut les tenir vivants dans de petits aquariums pendant quelques jours si l'on prend la précaution de renonveler l'ean de temps en temps.

Les colonies de cette espèce présentent, plus que tous les autres IIydroïles, le phénomène du polymorphisme. Elles se composent toujours de trois sortes d"individus ou piutôt de trois formes de zooüles.

$1^{\circ}$ Les Polypides ou Hydranthes, zoö̈les mangeur's qni portent une courome de tentacules disposís rin me ou en deux rangées.

2" Les zooïdes qui donnent naissance aux sporosacs. Ces individus chargés des soins de la reprodnction sont appelés gonosomes par Allman. Ils sont plus grciles et plus courts que les Hydranthes; ils sont dépourvus de tentacules; quand il sont complétenent déreloppés, ils portent un grand nombre de sporosacs (I) ì différents états de dereloppenent.

नo Strethill Wright a décrit me troisième sorte de roö̈des : certains individus dépourvus à la fois de tenta-

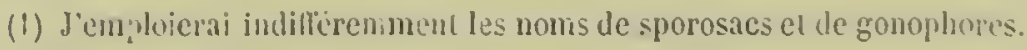


cules et de bouche ne donnent janais naissance à des sporosacs. Ils ont un corps allongé de forme cylindroïde. J'ai constaté que leur système musculaire est extrêmement développé, principalentent près de leur base d'insertion; c'est graice à cette particularité qu'ils s'enroulent dès qu'on les touche. Je ne les ai jamais vus former une spirale à plusieurs tour's comme Wrighı et Allman en ont liguré : tout au plus décrivent-ils, quand ils sont emroulés, un cercle complet ou plutôt un tour de spire. Ils se rencontrent exclusirement près des bords de la colonie.

Je n'ai pas observé la quatrième forme de zooïles décrite par Strethill Wright et Hinclis. Des filaments allongés semblables à de longs tentacules et dépourvus de la faculté de s'enrouler en spirale ont été considérés par' ces naturalistes comme représentant une quatriène catégorie de zooïles. Allman a recomnu que leur présence n'est pas constante. Quant aux sporosacs déreloppés directement sur le cœnosare, je ne les ai jamais observés, et en cela je n’ai pas été plus lieureux que Allman. Je crois comme lui que Wright a commis une erreur en regardant comme des sporosacs nés directement sur le coenosarc des zooïdes ai blistostyle très-court.

Je ne moccuperai ici que des zooïdes de la seconde eatégorie : les gonosomes ou zooïdes reproducteurs.

Les IIỵdractinies sont dioïques : tous les zooïdes rejproducteur's d'une même colonie sont tonjour's du mème sexe.

On trouve dans une même colonie dè zooïdes de tout dige et de toutes dimensions. Il en est qui ne portent encore aucune trace de gonophores à côté d'autres qui en sont fortement chargés; de plus, chaque gonosome porte des sporosacs à des états de déreloppement extrêmement 
différents. Ces deux eirconstances sont éminemment arantagenses pour l'étude de la question de l'origine des produits sexuels. Quand on a sous les yeux une colonie màle, on est sùr en prenant n'importe quel zooïde reproducteur d'avoir clıoisi un individn inâle et il n'est pas nécessaire de recourir à l'exameı des organes et des produits sexuels pour déterminer le sexe. On peut done affirmer que tel gonosome chez lequel les sporosacs n'ont pas encore commencé à se former, produira des sporosacs mâles on femelles; il est possible de déterminer arec certitude le sexe de l'organe reproducteur dès le moment de son apparition et bien avant qu'on puisse le distinguer par la nature de ses produits.

Les colonies femelles se reconnaissent avec la plus grande facilité. Le vitellus des œufs cst d'un bean rouge et comme les œufs se laissent apercevoir à travers les parois minces et transparentes des sporosacs, ceux-ci apparaissent avec cette même couleur vive qui caractérise ì première vue les colonies femclles. Les testicules, au contraire, sont d'un blane laiteux et l'ensemble de la colonic présente ì peine une faible teinte roséc qui dépend de la présence d'une matière pigmentaire rose dans la cavité digestive et dans les cellules de l'endoderme. La teinte générale de ces colonies màles est plus ou moins marquée; clle varic entre le blane presque pur et le rose plus ou moins foncé, ponvant même passer an brun dans quelques colonies. Je ne sais pas ì quoi il faut attribuer ces différences de teinte.

Caractères communs aux zooüdes reproducteurs mîles et femelles.

Les zooïdes reproducteurs se distinguent immédiatement des Hydranthes par l'absence complète de tenta- 
cules. Ce caractère avait été reconnu par mon père, quand il fit dans son mémoire sur les Tubularides de la còte d'Ostende la description de son genre Hydractinie (I). De Quatrefages, qui décrivit quelque temps après, sous le nom de Synhydra, le même animal, avait aussi signalé cette différence entre les individus reproducteurs et les individus mangeurs (2).

Cependant il cxiste autour de l'extrémité supérienre (pôle oral) du zooïde des tubercules globulaires qui ont l'air de tentacules atrophiés. Ils donnent à cette extrémité du corps une certaine ressemblance avee un chon-fleur (dc Quatrefages). C'es tubercules sont exclusivement formés par l'ectoderme et jamais l'endoderme ne leur envoic aucune espèce de prolongement, ce qui les distingue des tentacules proprement dits. Ces tubercules sont disposés en deux séries alternantes et leur nombre varie avec l’àge du zooïde. Ils ont les mèmes rapports de position avec la bouche que les tentacules chez les Hydranthes. Ces tubercules sont toujours fortement chargés d'organes urticants, comme les rrais tentacules des Hydroïdes. On pourrait les appeler des pseudotentacules.

Je crois en effet que ces tubercules ne sont que des tentacules dégénérés (généalogiquement parlant) et je pense que les zooïdes reproducteurs ne sont dans le mêne sens que des Ifydranthes modifiés à raison du ròle plìsiologique particulier qu'ils ont à remplir dans l'écononnic de la colonie. Dans le cours de l'évolution ontogénique de ces

(1) P.-J. Van Beneden, Recherches sur l'embryogenie des Tubulaires, MÉM. DE L'ACAD. RoY. de BELG, 1. XVIl.

(2) De Quatrefages, Mémoire sur la Synhydre parasite, Axx. sc. xat., 20 sér., t. XX, [. 2.53. 
zooïdes, les tentacules proprement dits n’apparaissent plus. Les pseudotentacules se montrent dès le début, aree leur caractère de simples dépendances de l'ectoderme.

Le corps des zooïles reproducteurs a une forme cylindroïde. On peut y distinguer quatre régions (p). 1, fig. 2, Je( 4) :

1. Une portion basilaire toujours plus large et présentant dans tonte sa liauteur le même diamètre, je l'appellerai la région gastrique; $2^{\circ}$ une portion moyenne aux dépens de laquelle se développent les sporosacs, je la lésignerai sous le nom de région germinatice; $\breve{J}^{\circ}$ une portion terminale plus grèle que la région basilaire dans laquelle la eavité digestive est très-étroite, je l'appellerai région cambiale; $4^{\circ}$ un renflement terminal dans lequel le canal digestif se dilate en une cavité ovoüdle. C'est cette partie terminale renflée qui porte les pseudotentacules et l'orifiee buecal.

La présence de cel orifice a été niée par de Quatrefages; Allman doute aussi de sa présence (1). Cependant Strethill Wright avait déjjà remarqué que la moindre pression exercée sur les parois du corps du zoö̈de détermine l'évacuation par un orifice terminal des matières alimentaires (2). Agassiz. a trouvé une bouche bien développée chez l'espèce amériraine du genre IIydractinic (3). J'ai pu m'assurer de l'existence constante d'un orilice à l'extrémité du renflement

(1) Monography of the Tubularian Mylroïls, part. II, p. 232-3. "I have not sitisfied mysell as to the complete alssence of a mouth in the blastostyle.

(2) Strechill Wright, Proc. roy. phys. Soc Edimb., 1862.

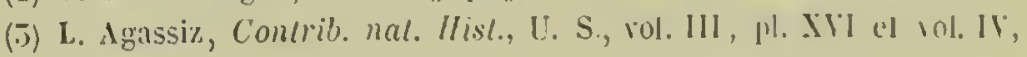
1). $2 \pm 7$ 


\section{( 21 )}

terminal des zooïdes reprodueteurs mâles et femelles. Cet orifice est petit et sert probablement d'anus plutôt que de bouche, quoique primitivement il ait rempli l'me et l'autre fonction. Il a cessé de jouer le rôle d'orifice buceal, it la suite de l'atrophie des tentacules et il est devenu très-étroit, depuis qu’il ne sert plus qu’ì expulser des résidus peu volumineux.

Le corps tout entier des zooïdes reproducteurs est formé, comme chez tous les Hydroïdes, d'un cctoderme et d'un endoderme; ces deux couclies eellulaires sont en continuité l'une avee l'autre au niveau de l'orifice lunccal. L'endoderme et l'ectoderme sont formés dans toutes les régions du corps d'une seule couche de cellules; mais ces cellules, surtout celles de l'endoderine, ont des caractères fort différents dans ces diverses régions. Je n’ai à m'occuper dans ce travail que de la région germinative; je déerirai plus loin les earactères qu'affectent dans les deux sexes ces deux eouches cellulaires.

Entre les deux conches existe nne membrane sans structure dont l'épaissemr varie d'un point à un autre (Stützlamelle de Leydig et de Reichert). La face externe de cette membrane n'est jamais lisse; au contraire, elle est toujours striée longitudinalement. Elle est tapissée dans toute la longueur du corps par une couche de filsres musculaires. d'ai pu démontrer chez les Hydractinies la continuité des cellules de l'ectoderme avec ces éléments museulaires et confirmer pour ec genre les belles observations de Köllilier et surtout de Kleinenberg sur l'Hydre d'eau (louce.

Les procédés qui m’ont le mieux réussi pour isoler ces éléments ont été: $1^{\circ}$ la macération pendant 24 lieures de 
colonies traitées au préalable par une solution de $\frac{1}{i 000}$ d'acide osmique el $2^{\circ}$ Ie traitement prolongé par une solution de $\frac{1}{400}$ de chlorure de platine mêlé a vec $\frac{1}{400}$ d'acide chromique; cette méthode m’a donné d'excellents résultats. A près un séjour de deux à trois jours dans celte solution, les Hydractinies laissent isoler facilement les éléments constitutifs de l'ectoderme.

Je décrirai tout au long dans un travail spećcial sur l'organisation et le développement des Hydractinies mes recherches sur la structure et la formation de l'ectoderme. Je veux seulement en dire un mot ici.

La couche musculaire est surtout épaisse dans la région basilaire du corps; mais elle sétend partout entre l'endoderme et l'ectoderme à la face externe l'une lamelle sans structure. Elle est composée de véritables fibres-cellules. Chaque fibre consiste en un cordon de substance réfringente (substance musculaire) entouré d'une mince couche de matière protoplasmique dans laquelle on observe un noyau ovalaire.

La substance musculaire diffère notablement par son apparence, principalement par son lıomogénéité et sa réfringence des cellules de l'ectoderme. Par ces caractères, ces ćléments sont assez semblables aux fibrilles musculaires des Cordylophora, décrites par F.-E. Schulze; mais elles ont les bords lisses chez les Hydractinies. Les fibres musculaires des Hydractinies diffèrent beaucoup des prolongements musculaires de l'Hydre décrits par Köllilier et Kilcinenberg.

Aux deux extrémités de la fibre, la substance musenlaire s'amincit et se termine en pointe, tandis que la couche protoplasmique, au contraire, devient plus épaisse. Dans un grand nombre de préparations, j’ai ru la conti- 


\section{( 25 )}

nuité de ces fibres-cellules avec une cellule de l'ectoderme. Cette continuité s'établit par l'intermédiaire d'un cordon de protoplasme dans lequel on ne peut plus distinguer aucune trace de substance musculaire.

A côté de ces fibres, on en roit, dans toutes les préparations, une foule d'autres qui se terminent à une de leurs extrémités par un cordon protoplasmique; mais la continuité avec les cellules ectodermiques a été rompue par la dilacération. Les cellules ectodermiques représentent plyysiologiquement des cellules nerveuses faisant en même temps fonction de cellules de sens et de cellules centrales. Les cordons protoplasmiques qui les relient aux fibres musculaires sont de véritables nerfs moteurs. L'ectoderme fait fonction de système nerveux; à ses dépens se forme le feuillet musculaire arec lequelil reste en continuté. Mes observations confirment en tous points les belles recherches de lileinenberg sur l'Ilydre d'eau douce, et je ne puis que me rallier aux considérations qu'il a énoncées avec tant de talent dans son remarquable mémoire. Je ne puis admettre cependant l'absence d'un épithelium cutané; les faits embryogéniques sur lesquels Ki lcinenberg s’appuie pour dénier aux Ifydrö̈des un système épidermique ne me paraissent pas concluants. A mon avis, l'ectoderme représente ì la fois tant au point de rue anatomique qu'au point de vue physiologique, l'épiderme et le système nerreux confondus. Lce seul point important par lequel mes observations sur les Hydractinies diffèrent des siennes, concernent la composition histologique des èléments musculaires. Chez l'Hydre il n'existe que des fibrilles musculaires qui sont de simples prolongements des cellules ectodermiques, de sorte que l'ectoderme est formé de véritables cellules neuromusculaires (Kileinenlorg). Chez les IIydractinies la complica- 


\section{( 24 )}

tion histologique est plus grande. La cellule neuromusculaire s'est divisée en cellule neuroćpithéliale, en fibre nerveuse et en fibre musculaire (cellnte musculaire). Allman a trouvé des fibres musculaires longitudinales entre l'endoderme el l'ectoderme des tentacules de la Tubularia indivisa. Il a reconnu que ces fibres sont pourveses d'un noyau ovalaire à mucléole fort brillant. (Allman, Monogrte phie, part. II, p. 206.)

J'ai pu constater aussi la continuité entre les cellules de l'ectoderme des bras des Méduses clicz les Campanulaires et les fibrilles d'une extrème ténuité qui sont immédiatement sousjacentes à l'ectoderme. Je crois, sans cependant en être bien certain, qu'il ı’existe pas chez ces petites Méduses de véritables cellules musculaires, mais senlement des fibrilles. Autour des sporosacs chez les Hydractinies, jai trouvé les éléments musculaires beanconp plus grèles qu'autour de la région basilaire du corps et je n'ai pas pu me convaincre de l'existence de noyaux cellulaires dans les éléments musculaires de cette région. F.-E. Schulze n’a pas trouvé de fibrilles musculaircs autour des gonophores des Cordylophora.

J'én viens maintenant à la description de la région germinative du corps des gonosomes. Cette élude est inséparable de la description des sporosacs el de leur développement. Mais il sera nécessaire de faire successirement cette étude chez les gonosomes qui portent des sporosacs femelles et chez ceux qui donnent naissance à des produits màles. Pour simplifier le langage, je les appellerai simplement gonosomes femelles et gonosonies màles. 
Mélhodes de préparation.

Avant de faire l'étude des caractères de l'ectoderme el de l'endoderme dans la région germinative, il est nécessaire d'exposer les méthodes que j’ai employées.

Les différentes couches cellnlaires de ces organismes sont si transparentes et si nettement délimitées, que, dans la plupart des cas, il suffit d'examiner le gonosome vivant dans l'ean de mer. On enlève au moyen d'un 'scalpel une partie du coenosarc et on la porte sous le microscope simple. A u moyen d'aiguilles ou d'un scalpel, on détache de sa base d'insection l'individu que l'on veut examiner. Si l'on a rénssi à l'isoler sans le blesser, et si l'on prend la précaution de le recourrir d'un conrre-objet sans trop le comprimer, le jeune gonosome s'allonge bien tôt et s'épanouit absolument comme s'il se trourait encore fixé sur son polypier; il se laisse alors examiner mème arec les plus forts grossissements. On peut mème, en procédant lentement et progressivement, exercer sur lui une certaine pression, sans qu'il se contracte. Si l'on veut étudier la région germinative et le développement des sporosacs chez $11 n$ gonosome adulte, il est indispensable d'enlever successivement arant de le porter sous le microscope tons les sporosacs que l'on peut apercevoir à la loupe, en commençant par les plus déreloppés et en procédant d'arrière en avant. Les sporosacs se détachent très-facilement, dès que l'on comprime leur pédicule d'insection au moyen d'une aignille. Quand le gonosome se trouve ainsi réduit à son blastostyle et à quelques sporosacs en voie de développement et à peine perceptibles il la loupe, on peut l'examiner sous le microscope et cette mutilation ne l'empêche pas de s'épanouir absolument 


\section{( 26 )}

comme s'il n'avait pas souffert le moins du monde de l'opération qu'on lui a lait subir.

Je me suis servi avec grand avantage, principalement pour l'étude des détails lisstologiques, de différents réactifs, et jai en recours à différentes métlodes pour durcir, colorer, couper et dilacérer. De tous les réactifs que j’ai employés, celui qui m’a donné les meilleurs résultats, c’est l'acide osmique en solution faible. Je l'ai employé de la manière suivante: quand j’arais étudié un individu virant après l'avoir préparé comme je l'ai dit ci-dessus, je faisais agir sur lui sous le microscope une goutte d'acide osmique (en solution de $\frac{1}{100} \dot{a} \frac{1}{600}$ ) que je laissais filtrer peu à peu sous le courre-oljet. Je pouvais observer ainsi l'action progressive du réactif sur les tissus qui, sous son influence, ne perdent rien de leur transparence primitive, mais qui se colorent légèrement et dont certains éléments deviennent d'une netteté admirable. C'est le cas plus particulièrement pour les cellules de l'ectoderme et de la couche musculaire. Ce réactif agit moins avantageusement sur les cellules de l'endoderne et sur les œufs. Ceux-ci noircissent trop rapidement à raison de la quantité de substances grasses qu'ils renferment. Quand le réactif a suffisamment agi, on lave après aroir enlevé l'acide par aspiration.

La macération pendant 24 heures, après traitement par une solution d'acide osmique de $\frac{1}{1000}$, m'a permis d'isoler les cellules de l'ectoderme et de démontrer leur continuité avec les fibres musculaires. Je me suis servi arec grand avantage dans le mème but d'une solution de $\frac{1}{400}$ de clılorure de platine mêlé à $\frac{1}{400}$ d’acille clromique. Pour l'étude de l'endoderme je me suis trouvé très-bien d'une solution extrèmement diluée d'acide acétique. Quant aux cellules de lectoderme, elles gonftent et deviennent tout ì fait 
transparentes sous l'influence d'une solution même trèsfaible de cet acide. J'ai bien réussi à isoler les cellules de l'endoderme après une macération de denx à trois jours dans le liquide de Müller ou dans l'acide cluromique à $\frac{1}{1000}$. Les méthodes ordinaires de coloration par le carmin et le picrocarminate ne sont pas recommandables: l'ectoderme ne se colore presque paset il s'altère rapidement au contact d'une solution alcaline. Les cellules de l'endoderme, au contraire, se cliargent tellement de carmin qu'en quelques instants elles deviennent tout à fait opaques. Par contre j'ai obtenu d'assez bonnes préparations par le rouge d'anhiline en solution très-faible et par l'hœmatoxiline.

\section{Gonosomes femelles.}

Comme je l'ai fait olsserver plus haut, on trouve dans une même colonie des gonosomes à tous les degrés de déreloppement : il en est qui portent un grand nombre de sporosacs: les uns, insérés à la face externe de la région germinative, sont les plus jennes; les autres, au contraire, sont fixés par un pédicule grêle à la partie supérienre de la région gastrique. D'autres gonosomes beaucoup plus petits portent à peine un ou deux sporosacs (pl. I, fig.5 et 4) fort peu arancés dans leur développement; on en trouve, enfin, chez lesquels il n'existe encore aucune trace de sporosacs. C'est un semblable individı que j’ai liguré 11. I, fig. 2, et que je vais décrire en premier lieu.

Les caractères de l'ectoderme sont sensiblement les mèmes dans tontes les régions du corps. La description que je rais en donner ne s'applique done pas exclusirement à la région germinative.

Ectoderme. - On sait que Ecker arait nié l'existence 


\section{( 28 )}

d'éléments cellulaires différenciés dans l'ectoderme aussi bien que dans l'endoderme des Iydres. N. Reichert a soutenu qu'il n'existe ni chez l'Hydre, ni chez les Tuluulaires, ni chez les Campanulaires, aucune trace de cellules ni de noyaux de cellules dans l'ectoderme. On peut considérer comme définitivement établi, après les recherelies de Allman, de Leydig, d'Agassiz, de Huxley, de Claus, de F. E. Schulze et de Kleinenlserg que l'ectoderme de tous les Coelentérés est formé de cellules à noyaux. Il est à peine concevable que II. Reichert, qui doit posséder le maniement du microscope, et doit connaître les méthodes employées aujourd'hui en histologie, ait pu émettre une scmblable opinion. Il n'est pas un des naturalistes que je riens de nommer qui ne soit tout aussị capable que M. Reicliert de distinguer un organe urticant d'un noyau de cellnle.

En n'importe quel point du corps de l'Hydractinie, on distingue nettement que l'ectoderme est formé de cellules dont les noyaux se reconnaissent chez le gonosome vivant, sans l'addition d'aucun réactif. Les circonscriptions cellulaires sont plus ou moins distinctes: quand le zooïde est à moitié contracté, le bord externe de l'ectoderme est ondulé; tout le feuillet externe est alors couvert de petites bosselures détcrminées par les cellules de l'ectoderme qui, dans cet état de demi-contraction du gonosome deviennent convexes. Quant, au contraire, le gonosonic est étalé, ces bosselures disparaissent plus on moins complétement : les cellules extodermiques deviennent planes et ressemblent alor's à des cellules endothéliales.

Sons l'influence de l'acide osmique, les contours cellulaires apparaissent avee une netteté admirable; on voit alors tout l'ectoderme formé de cellules polygonales convexes ì leur face externe, et séprarées les unes de autres 
par des sillons plus ou moins profonds (pl. I, fig. ö et 6). Après la macération dans l’acide osmique, dans le liquide de Müller ou dans le mélange de chlorure de platine et d'acide chromique, ces cellules peuvent être facilement isolées. Leurs noyaux se colorent, si l'on traite par une solıtion faible d'anhiline ou d'lırmatoxiline.

L'épaisseur de ces cellules et de la couche ectodermique rarie beaucoup. J'ai trouvé, sous ce rapport, d'un individu ̀̀ un autre, des différences cxtrèmement notables, et en une mème r'égion du corps, d'après l'épaisseur de la couche, ces cellules présentent des caractères assez différents. Le noyau est toujours de forme sphlı́rique à contour's assez pâles quoique toujours très-nets; il est toujours ponrvu d'un nucréole réfringent. Quand l'ectoderme est mince, les cellules ne présentent extérieurement qu'une très-laible convexité (pl. I. fig. ¿̈); leur contenu est partout granuleux, comme s’il était exclusivement formé par de la matière protoplasmique. Au contraire, quand l'ectoderne est épais, la surface c.rterne des cellules est très-convere: elles renferment alors une ou plusieurs vacuoles remplies d'mn liquide homogène et parfaitement transparent (pl. I, fig. 6). Alors le noyau est refoulé à la périphéric avec la matière protoplasmique dans laquelle il se trouve toujours logí.

Dans l'ectoderme de la l'égion germinative aussi lien qu’a la surface des sporosacs, il n'cxiste qu'un fort petit nombre de corps urticants. On en trouve un çà et là, habitucllement logé dans une cellule d'une forme particulière et située un peu plus profondément que les cellules ordinaires de l'ectoderme. Ces cellules reif ferment en outre un noyau fort petit toujours situć près de la lamelle basilaire à còté du corps urticant. Je n’ai pas constamment rencontré ce noyau. Quelquefois cependant ces cellules attei- 


\section{$(50)$}

gnent la surface de l'ectoderme, et alors elles présentent habitucllement un petit prolongement filiforme. Allman, Ehrenberg, Leydig, IIæckel, Schulze et Kleinenberg ont attiré l'attention sur ces petites soies (Spitzen IIürchen). Allman les considère comme des organes de toucher, ce qui me parait fort peu probable; il les appelle palpocils. Cependant je les ai plus fréquemment rencontrés sur les tentacules et les pseudo-tentacnles que sur le reste de la surface du corps. Je crois a voir trouvé aussi quelquefois des organes urticants logés dans des cellules ordinaires de l'ectoderme. Probablement ils y arrivent secondaircment.

Dans la région germinative du corps aussi bien qu'iu la surface des spornsacs, l'ecloderme est loujours formé d'une seule couche de cellules. Les quelques rares cellules qui donnent naissance aux corps urticants, et qui occupent la partie profonde de l'ectoderme, ne constituent jamais une couche continue de façon à former un tissu comparable au tissu intersticiel de l'Ifydre (K'leinenberg) ou mème à la couche profonde le l'ectoderme du Cordylophora (F. E. Schulze). Le tissu intersticiel n'est représenté chez les Hydractinies que par quelques cellules disséminées çà et là dans lesquelles se forment les corps urticants. Je ne décrirai ici ni les caractères ni le mode de formation de ces derniers organes, d'abord parce que leur étude est difficile à cause de leur petitesse et que je n’ai pas pu les étudier suffisamment; en second lieu parce qu’ils ne présentent aucme importance eu égard à la question que jai principalement en rue de traiter dans ce mémoire.

Couche musculaire. - Quand on examine la coupe optique d'un gonosome vivant, on distingue nettement 


\section{( 51 )}

entre l'ectoderme et l'endoderune une couclie tout ì fait transparente qui parait fort réfringente et limitée par un contour plus foncé et moins régullier di còté de l'ecto-

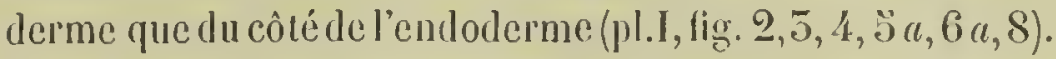
Cette couche est notablement plus épaisse dans la réggion basilaire du corps que dans la partie germinative. Elle est forméc à la fois par unc lamelle sans structure (Stïtzlamelle) et par des fibrilles musculaires accolées à sa facc externe. KRleinenberg donne à cette lamelle de l'Hydre le nom de lamclle musculaire. Il troure les prolongements musculaires des cellules de l'ectoderme engagés dans unc substance homogène et transparente qu'il appelle Bindemittel. Reichert avait observé cette même membrane chez l'Hydre; mais il la décrit comme une membranc sans structure, et il affirme que les prétendues fibres musculaires de l'Hydre ne sont qu'une illusion produite par un plissement que subit cette membrane pendant la contraction de l'animal! F. E. Schulze décrit chez le Cordylophora une lamelle hyaline (Stiitzlamelle) et des éléments musculaires appliqués à sa face externc. Chez les Hydractinies il n'est pas difficile de séparer complétement les éléments musculaires de la lamelle sans structure. Si l'on examine le gonosome de face, dans la région germinative, on aperçoit une striation longitudinale déterminée par des éléments situés sous les cellules ectorlermiqques, et qui ne sont autre chose que les éléments musculaires. Ils sont disposés parallilement les uns aux autres sans cependant se toucher mutuellement, au moins daus la partic antérieure du corps: la couche musculaire n'est continue ni dans la région germinative, ni dans la région cambiale, ni surtout à la surface des sporosacs. J’ai décrit plus lıaut les caractères des fibres musculaires dans la région basilaire du corps, et 
j’ai indiqué en mème temps les méthodesque j’ai employées pour isoler ces éléments. Dans la région germinative les fibres musculaires sont plus grêles, et je n’ai pas réussi à démontrer leur continuité arec les cellules de l'ectoderme. Cependant je ne doute nullement de l'existence de celte continuité. Kleinenberg dit qu’il a pu assez facilement isoler chez l'Hydre les cellules de l'ectoderme qui recourrent la surface des tubercules sexucls et montrer leur continuité avec les fibrilles musculaires. II m’a été impossible d'ohtenir ce résultat chę mes IIydractinies. Ce n'est que pour les fibres musculaires de la région gastrique et principalenent clez les individus spiraloüdes que j’ai pu démontrer celte continuité en isolant les fibres musculaires. Après la macération dans le chlorure de platine on parvient à isoler les filrilles musculaires et les cellules de l'ectoderme des sporosacs; mais je n'ai pas réussi à les trouver en continuité. Les fibres musculaires de la surface des sporosacs sont encore plus grèles el plus pâles que celles de la région germinative. Elles sont faciles à observer sur de jeunes sporosacs. Elles sont étirćes en pointe au moins à une de leurs extrémités. de n’ai pas trouvé, accolés aux fibres musculaires des sporosaes, des noyaux que je pusse considérer comme appartenant aux éléments musculaires. Mais la substance réfringente de la fibrille musculaire se montre entourée d'un peu de matière granuleuse (protoplasme). Ces fibrilles ue présentent aucnne trace de striation transiersale.

Lamelle basilaire. - Sous la couche musculaire se trouve une membranc sans structure tout à fait transparente et incolore. Elle a une épaisseur assez notable et peut être très-facilement isoléc, après macération, mème 


\section{$(55)$}

sur une assez grande étendue (pl. II, tig. 仓े). Quand on a enlevé complétement les cellules de l'ectoderme et les filrilles musculaires, on distingue encore à la face externe de cette membrane liyaline une striation longitudinale souvent très-marquée; celte face n’est pas lisse, ce qui se roit bien quand, après l'avoir isoléc, on la replic sur ellemème; le bord du pli montre alors des irrégularités. Je pense que la striation longitudinale de la membrane isolée et les irrégalités de sá sinrface dépendent de ce que la unaticre protoplastique qui entonre plus on moins complétement les filres musculaires reste en partic accoléc à la surface externe de la membrane. L'existence de cette lamelle sans structure a été signalée depuis longtemps che\% l'IIydre par Lcydig, plus récemment par Reichert, par F.-E. Schulze et par Kileinenberg. Reichert l'a trouréc aussi chez les Campanulaires; F.- E. Schulze chez les Corlylophora. Il est probable qu'elle ne maneque cliez aucun Iydroïle.

Endoderme. - Comme le montre la figure 2 (pl. I), la cavité digestive est assez large dans la portion basilaire du gonosome; elle présente à peu près dans tonte la hauteur le cette partic du corjus lo mème diamètre. Dans la régrion germinative la cavité digestive consiste en un entonnoir renversé dont le sommel correspond à pen près à la limite supérieure de cette régrion (pl. I, fig. 1, 2, 5 et 4) et marque le commenecment de la partic cambiale dn gonosome.

Liendoderme présente à peu près partout la même épaisseur; mais les caractires des cellules qui le conslifucnt varient beaucoup d'une région à l'autre.

Ilans la région cambiale du corps les cellules ont une lurme prismatique, mais leur largeur est extrêmement faible 


\section{( 34 )}

comparativement à leur hauteur (pl. I, fig. 9). Elles sont fortement serrées les unes contre les autres. Leurs limites sont difficiles à apercevoir; leur noyau clair est très-petit et plus on moins rapproché de la base d'insertion de la cellule sur la lanclle hyaline. Ces cellules sont formées d'un corps protoplasmatique finement, mais uniformément granuleux. On n'y trouve jamais ancune trace ni de vacuole, ni de liquide hyalin, ni de globules réfringents, ni de matière pigmentaire d'aucune sorte. Mais claque cellule porte à son extrémité un cil vibratile presque toujours unique. J'ai vu cependant quelques cellules isolées qui cu portaient deux. Ces cils sont fort longs: ils ressemblent beaucoup au filament flagelliforme des Flagellates.

Les cellules de la portion basilaire ou gastrique sont également de forme prismatique; elles ont à peu près la même hauteur que celles de la région cambiale, mais elles sont notablement plus larges (fig. T). Elles renferment un noyau plus volumineux, sphérique, à contour's très-pâles et toujours pourvu d'un nucléole. Ces cellules sont beaucoup plus claires; elles renferment soit une grande vacuole remplic de liquide hyalin, soil plusicurs vacuoles anastomosées. Le noyau est alors refoulé à la périphérie: il se trouve dans la paroi protoplasmique de la cellule; ou bien il est situé plus ou moins ilans l'axe de la cellule et de sa surface partent des filaments protoplasmiques. Dans la partic de la cellule qui circonserit immédiatement la cavité digestive le protoplasme est toujours fortement clargé de globules réfringents, peut être formés d’une matière grasse, et de grumeaux plus ou moins volumineux d'une matiere pigmentaire granuleuse. Chacune de ces cellnles est pourvue aussi d'un long cil flagelliforme.

Dans la région germinative, l'endoderme possìle à peu 


\section{$(5 \ddot{3})$}

près la mème épaisscur que dans la région cambiale. Ce qui frappe tout d'abord quand on examine ectte partic du gonosome, c'est l'existence dans l'épaisscur de l'endoderme de très-gros noyaux (fig. $1 a, 2, \overline{5}, 4 \mathrm{et} 8$ ) tout à fait transparents, homogènes, et qui ne renferment aucune granulation, mais seulement un nucléole rolumineux formé d'une substance très-réfringente. Dans le nucléole on trouve quelquefois, principalement dans les plus gros, une vacuole remplic d'une substance moins réfringente. Ces noyaux sont renfermés dans des cellules dont le corps fiusiforme se termine en pointe du côté de la cavité digestire et repose par une base élargie sur la membrane auhyste (fig. 8). Le corps protoplasmique de ces cellules est fort développé; il se constitue d'un protoplasme trèsfinement, mais uniformément granulcux.Ces cellules d'une forme si particulière et dont les noyaux sont tellement distincts qu'ils attirent tout d'abord l'attention quand on jette le regard sur un de ces jeunes gonosomes, sont séparées les unes des autres par des cellules prismatiques grêles toutes senblables à celles qui constituent l'endoderme dans la région cambiale du cor|ss. Elles en diffèrent sculement en ce qu'elles renferment souvent deux ou plusicur's noyaux. Ces cellules sont toutes pourves d'un long cil dirigé vers le pôle oral dı gonosome et vibrant arec une grande activité. C'est dans l'entonnoir qui termine supérieurement la cavité gastrique que les mourements ciliaires sont les plus apparents. Ces cils semblent très-rapprochés parce que les cellules qui les portent sont fort étroites. Les mourcenents ciliaires qu'on distingue dans l'entennoir rappellent tout à fait ceux des membranes ciliées des animaux supérieurs ou tout spécialement ceux que l'on olserve dans le tube digestif des Bryozoaires. 


\section{( 56 )}

Les grands noyaux des ecllules de l'endoderme, caractéristiques de la région germinative, atteignent leurs dimensions maxima au milien de cette région ( fig. 2, 5,4 et 8 ). Ils diminuent de volume au voisinage de la région canbiale di gonosome, ed, à la limite, il n'est pas possible te les distinguer des cellules prismatiques étroites de Pendoderme de celte région. On troure toutes les transitions entre les petits noyaux des cellnles prismatiques grêtes et les grands noyaux que j’ai décrils. D’un autre còté, à la limite de la région gastrique du gonosome, les cellules prismatiques étroites el dépourves de matières pigmentaires et de gololules réfringents, passent insensiblement anx grandes cellules qui composent l'endoderme de cette portion du corps.

Tels sont les caractires de la région germinative des jeunes gonosomes avant l'apparition des premiers sporosaes.

Comme je vais le montrer, c’est aux dépens de cette région que se forment les organes reproducteurs: ils ue sont à leur début que de simples diverticules des parois du corps du polype. Si l'on examine un jeune gonosonie, comme celui que jai figuré (pl. I, fig. 5 ), on le troure construit alsolument de la mème maniere que celui que je viens de décrire, avec celte senle différence qu'il porte sur ses parois latérales, dans la région germinative, un tubercule conoïle (b) formé à la fuis par l'endoderme el par l'ectoderme. Les caractères de ces deux couches cellulaires sont identiques dans le tubercule el dans toute létenduc de la région germinative. L'ectoderme y est seulement un peu plus mince: les cellules de cette couche sont un pen plus aplaties; mais ces différences sont si insignifiantes que je ne les aurais probablement pas aperçues, si elles ne se mapquaient davantage dans les tubercules plus développés 


\section{$(5 \bar{T})$}

(fig. 1). L'endoderme présente dans le tubercule qui n'est autre chose qu'un sporosac en voie de développement, la mème epaisseur que sur tout le reste de la surface du corps. Les cellules à grands noyaux ne sont que de jeunes reufs interposés entre les celtules épithéliales prismatiques de l'endoderme. Ces jeunes oufs se distinguent de ce qu'ils étaient dans la région germinative en ce que le noỵau est devenu un peu plus grand; le nucléole laisse voir une tache claire (Schörnche Korn) comme si sa substance foncée présentait une vacuole remplie d'un liquide moins réfringent; ces jeunes oufs s'insèrent sur la lamelle basilaire par une base élargie (fig. 11); leur extrémité interne n’atteint plus, au moins chez ceux qui se trouvent dans le voisinage de l'estrémité du diverticule, la limite interne de l'endoderme. Les cellules épithéliales prismatiques adjacentes à la cellule deveme ouf, se touchent à leur sommet de façon à recourrir celui-ci à son extrémité interne. L'ouf commence à étre expulsé de l'endoderime et refoulé entre celui-ci et la membrane sans structure qui le sépare de l'ectoderme.

A la coupe optique du tubercule on distingue dans ses parois quatre jeunes œufs (fig. 5 ). Dans le jeune sporosac pénètre un diverticule du tube digestif qui a une forme conoïlc ì sommet externe.

Dans le gonosome représenté (pl. I, fig. 4), il existe trois sporosacs inégalement développés. Le plus petit est situé dans un plan supéricur, plus voisin de l'extrémité orale du corps. Le moyen situé de l'autre còté du tronc est inséré un pen plus bas; le troisième notablement plus déreloppé se trouve fixé par un pédicule en un point plus éloigné encore du sommet de l'entonnoir qui termine supérieurement la cavité digestive proprement dite. 


\section{$(\overline{3} 8)$}

Le plus jeune sporosac a la forme d'un simple mamelon; il n'est qu'un diverticule des parois de la région germinative; il répond exactement à la description que je viens de faire du seul tubercule sexuel développé sur les parois du gonosome représenté pl. I, fig. 5 .

Le sporosac moyen (fig. 4 et 6 ) a une tout autre forme. Le tubercule primitif s'est renflé à son extrémité supérieure; il affecte maintenant une forme ellipsoïdale à grand axe plus ou moins perpendiculaire à l'axe dn corps du gonosome. Un étranglement encore peu marqué teud à se produire à la base du sporosac et à le séparer du corps du polypule. Les parois du sporosac sont toujours constituées par les deux couches fondanentales : l'ectoderme et l'endoderme. L'ectoderme est un peu plus mince, sauf it l'extrémité du sporosac où il s’éléve en un tubercule assez étendu dans lequel il est fort difficile de distinguer les limites des cellules (pl. I, fig. 18).

Les ovules sont encore logés dans l'endodernıe; mais ils se sont arrondis, surtout du côté interne. Ils ont grrandi: non-seulement leur corps protoplasmique, mais aussi leur résicule germinative et leur nucléole sont plus volumineux et plus granuleux; ces éléments restent toujours extrèmement distincts, et leurs caractères ne se modifient guère. Les œufs situés dans l'endoderme à la base du sporosac n'ont pas subi ces modifications (pl. I, fig. 20).

Le diverticule de la cavité digestive est devenu une simple fente (pl. I, fig. 4).

Il existe au sommet du jeune sporosac, sous l'celoderne, un organe à bords nettement marqués, qui apparaît sous la forme d'un croissant, dont la concavité s'applique immédiatement sur l'endoderme, tandis que par un point de sa 


\section{( 59 )}

surface convexe, il touche à l'ectoderme. La plus grande partie de son bord convexe en est séparée par une minee couche cellulaire. II fant recourir à l'examen de plus jeunes sporosaes pour connaitre le mode de formation de cet organe et pour arriver à en déterminer la signification.

Dans celui que nous arons figuré pl. I, ligg. 12, on roit quiu sommet du jeune sporosac l'ectoderme donne naissance, à sa face interne, à un tubercule formé de quelques cellules d'apparence cylindrö̈le ou conoïde, dans lesquelles on distingue un oudenx noyaux. les cellules de l'ectoderme se sont multipliées sur place et le tubercule cellulaire, en se développant vers l'intéricur, a refoulé devant lui l'endoderme, qui s'est déprimé en ce point de façon à se mouler exactement sur le tubercule. Celui-ci présente, à son début, différents aspects que j’ai figurés (pl. I, fig. 12, 15, 16). Dans quel(que cas il résulte d'me véritable invagination de l'ectoderme (fig. $15 \mathrm{et} 15$ ). Le tubercule plus ou moins conoüde au début (fig. 15) prend en se déreloppant, par suite de la prolifération des cellules qui le constituent, une forme globulaire (fig. 12). Les cellules de l'endoderme, en se moulant sur lui, se glissent réritablement entre le tubercule et l'ectoderme de façon à former à ses côtés de's sortes de cornes ou plutôt des lames qui font l'effet de denx cornes, quand on les observe à la section optique. Le tulsercule se développe en largeur à son extrémité interne et s'élale sur' l'ectoderme en même temps qu'il se pédiculise de plus en plus (fig. 14 et suivantes). C'est alors quil prend la forme d'un croissant ou plutòt d'une soutcoupe appliquée sur l'endoderme par sa concavité. Un pédi cule le relie encore à l'ectoderme. Les cornes de l'endoderme se sont développées progressivement en se glissaut en quelque sorte entre le tubercule modifié dans sa forme 


\section{(40)}

et l'ectoderme proprement dit. Je donnerai ì ces cornes développées aux dépens de l'ectoderme le nom de lames médusoïdes; Ic bourgeon qui apparait à la face interne de l'ectoderme et dont je viens de décrire le développement, sera désigné sous le nom d'organe testiculaire. Je dirai plus loin pour quel motif j’ai choisi ces noms.

En mème temps que le bourgeon testiculaire se déreloppe et qu'il clange de forme, ses cellules et surtout les noyaux cellulaires se multiplient rapidement. Le tissu de l'organe testiculaire se caractérise toujours par l'abondance de ces noyaux assez volumineux, si on les compare à ceux des lames médusoïdes. Ils sont, d’abord de forme sphérique et assez semblables à ceux de l'ectoderme, sauf qu'ils sont plus petits.

Les lames médusoïles ou cornes de l'endoderme sont constituées par des cellules formées d'une substance homogène dans laquelle on distingue de tout petits noyanx arrondis. Au début, il est impossible de distinguer la limite inféricure de ces lames du còté de l'endoderme, aree lequel clles se trourent en continuité de substance (fig. 12, 15, 14 et 18).

Pendant le développement ultéricur des sporosacs, l'organe testiculaire s'élale progressivement en une lame qui recouvre une partic de plus en plus étendue de la surlace de l'endoderme. En mème temps que l'organe se développe en une lame testiculaire, il devient de plus en plus mince, et ses noyaux cellulaires, d'abord splićriques, deriennent ovalaires, puis successirement ils saplatissent de plus en plus. La lame testieulaire, en se développant, détermine l'extension progressive des lames médusoïldes qui se forment aux dépens des cellules de l'endoderme et que l'on tromse partont entre la lame testiculaire et la 
membranc lryaline; celle-ci reste accolée à la face interme de l'ectoderme (pl. J, fig. 21).

Dans un sporosac, complétement développé, on trouve, cu allaut de dehors en dedans : $1^{\circ}$ l'ectoderme; $2^{\circ}$ la couche de filses musculaires; $5^{\circ}$ la lamelle hyaline sans structure; $4^{n}$ la lame médusoïde; $5^{\circ}$ la lane testiculaire; $6^{\circ}$ l'endoderme. Le pédicule qui rélie primitivement l'organe testiculaire an feuillet ectodermique s'atrophie trèstòt. Les extrémités des cornes (lames médusoïdes) se toucheut alors et une lame cellulaire continue se trouve ćtalée entre la lame testiculaire et l'ectoderme (fig. 1, et fig. 20).

Allnan a étudié et décrit le déreloppement du sporosac des Hydractinies. Il prend nème ce développenent comme type pour l'ensemble des Tubularides. \&Au début, dit-il, les sprorosacs sont de simples tubercules creux, nés des parois du blastostyle et formés à la fois par l'endoderme el par l’ectoder'ne. Bientòt l'endoderme se troure séparé de l'ectoderme au sommet lu sporosac par l'interposition d'une petite masse granuleuse, aux dépens de laçuelle se forment les produits sexuels mâles et femelles. A ce moment lectoderme s’'st différencié en deux couches cellulaires et nous arons dès lor's tous les éléments dont se conslitue le sporosac enmplétement déreloppé. L'endoderme devient le spadix; celui-ci est entouré par les produits sexuels; linterne des deux feuillets de l'ectoderme devient l'endothèque; l'externe devient l'ectothèque.

"Daus les sporosacs d'autres Hydrö̈des, dit-il, il apparait entre l'endothèque et l'ectolhèque une membrane intermédiaire dans laquelle on observe des canaux gastrorasculaires (la mésothèque).

" Je n'ai jamais pu roir, dit Allman, comment se forme 
cette membrane; clle apparait toujours complétement déreloppée dès quon pent la reconnaitre "On te voit. ecs données sont complétement inexactes et MI. Allman reconnaît lui-mème l'iusuffisance de ses observations. L'existence de lorgane testiculaire dans le sporosac des Hydractinies lui a échappé et son exposé du mode de dérelopiement des parties du sporosac est tout different de ce que j’ai ınoi-mème observé. Les fiğures schímatiques qui ont été publiées par Gegenbancr, d'alıord dans l'Allas de V. Carus et ultéricurement dans son Traité d'anatomie comparée, pour représenter les homologies entre les gonophores des Hydroïles en général, ne donnent aucun renseignement relativement à l'origine des produis sexucls: Gegenbaucr a eu pour but d’établir les homologies cpüil arait constatées entre les sporosaces et les principales formes de Méduses; mais il n’a pas cherché à déterminer la signification des différentes parties de la Mléduse dans leurs rapports avec les organes de la génération et l'origine première de ces éléments lui a échappé.

Jc n’ai pas parlé jusqu’à préscnt d"une particularité inportante que présente constamment l’organe testiculaire, quand il a atteint la forme d'un croissant. $\Lambda$ ce moment il existe dans cet organe une fente horizontale, paraltèle à la face concave et à la face convexe de l'organe; les noyaux cellulaires sont disposés en deux couches, séparécs l'une de l'autre par cette fente: la conche profonde, adjacente à l'endorlerme, est homologne de l'ectoderme du manubrium des Mélnses el la conche externe adjacente aux lames médusoüdes représente la conche cetodermique du sous onbrelle des Héduses. Cette feute sétend rn mìme temps que l'organe testiculaire; quand celui-ci se troure réduit à n'être plus qu’une mince lame cellulaire, il devient inıos- 
silıle de la distinguer; peut-être même disparait-elle conplétement.

Dans quelques sporosacs j’ai vu la fente de l'organe testiculaire s'onvirir à l'extérieur à la surface de l'cetoderme (pl. l, fig. 15). Ceci montre que la fente testiculaire n'est que l'extension de la fente que l'on observe dans quelques tubercules tels que celui qui est représenté (pl. I, fig. 15), et qui elle-même u'est que le résul tat de la formation de l'organe par invagination. La fente testiculaire doit être considérée comme se formant primitivement par invagination de l'ectoderme, alssolument comme cela se produit pour la formation de la cavité digestive de la Gastrula. Ce mode de formation de la gastrula par invagination a été observé chez l'Amplhioxus, les Cýclostomes et certains Batraciens; les Sagitta, les Plıoronis et beaucoup d'autres vers; les Cyclas, Polycera, Eolis, Doris, Plcurobranchus, Arion et Limax, parmi les mollusques; chez plısieurs Échinides el Astérides parmi les Échinoder'mes, enfin clıez plusieurs Coelentérés tels que la Pelagia noctiluca, Agalma rubrum, certaines Actinies, enfin chez les Cténoplıores. Mais dans tous les types d'organisation ontrouve d'autres espèces chez lesquelles la gastrula, au licu de se développer à la suite de l'inragination d'une moitié de la vésiculc blastodermique, se forme par creusement d'une cavité dans un amas de cellules affectant primitivement la forme d'un ovoïde plein. D’après les observations de Van Bambelic, ce serait le cas chez le Pélohate; il en est de même chez beaucoup d'Annélides, d'Hirndinćes, le Trématodes; chez la plupart, si pas cliez tons les Arthropodes; chez beaucoup de mollusques; cliez tous les Spongiaires; enfin chez la phipart des Cœlentérés. Le procédé de formation d'une cavité prar cren- 


\section{(44)}

sement conduit au mème résultat que l'invagination el doit ètre considéré comme une simple modification de ce dernier mode.

L’invagination est le procédé primordial : on peut le démontrer facilement par l'élude comparative du mode de formation de la gastrula. On pent observer toutes les transitions entre une véritable invagination et un véritable creusement. On tronve encore une preuve de lidentite fondamentale de ces deux processus de développement dans l'élude comparative du mode de formation des organes nerveux centraux. D'après les récentes olsservations de Kiupper, Götte, Rieneck, OEllacher et d’autres la moelle. rúsulte, chez les poissons ossenx, d'un simple épaississement du fenillet externe; tandis que chez la plupart des vertébrés elle se forme, par invagination, aux dépens du mème feuillet.

L'étude du mode de formation du systeme nervenx central chez les Arthropodes, chez les mollusques et chez les Vers a conduit à la même conclusion.

Ray Lanliester, qui a altiré plus particulièrement l’atten. tion sur l’identité fondamentale de ces deux moles de développement d'une cavité interne, a fait connaitre qur chez certains mollusques l'otocyste se forme par unc réritable invagination du feuillet externe (Céphalopodes, Loligo), landis que clicz, l'autres le mème organe se forme par développement progressil d’un tubereule cellulaire pui pro. cèle du mème fenillet embryonnaire (Nudibranches).

De neme pour la formation de lorgane esticulaire cliez les Colentérés, le processus primordial a été l’invagimation. Mais secondairement le développement s'est simplifié et la cavité testiculaire s'est forméc par la production d'une simple fente dans un tuberculaire cellulaire primiti- 


\section{(40̈)}

rement massif; cette modification dans l'érolution est le' résultat de celte tendance qui sollicite le développement onlogénique d'un organe à devenir plus direct et plus simple que le développement phylogénique: l’ontugénie n'est qu'une simplification, nun alorégé, une histoire condensée de l'histoire phỵlogénique. Ceci est rrai surtout, s'il sagit d’un organe rudimentaire, comme l'organe testiculaire dans le sporosac femelle. Ce qui est remarquable, c'est que les deux procédés se présentent encore côte ì ròte dans les sporosaes d'une mème espèce, que dis-je, sur un mème gonosome d'Ilydractinies. Je dois ajouter, eependant, que le procédé du fendillement est beaucoup plus fréynent que l'invagination.

Ouelquefois l'organe testiculaire ne se péliculise pas. l.e bourgeon cellulaire de l'ectoderme s'étale dès le déhut et l'endoderme ne s'insinue pas entre le bourgeon testiculaire et l'ectoderme. Les lames médusoïdes manquent alor's, ct la lame testiculaire s'applique immédiatement ì la face interne de la lamelle hyaline sous-ectodermique. J'ai trouré quelquefois des sporosacs conslitués de cette manière chez les Hydractinies femelles (pl. I, fig. 16).

Dans l'autres individus l'organe testiculaire, au licu de se développer également dans tous les sens, se porte tout entier d'un côté; il est appliqué contre l'une des faces latérales de l'endoderme. De l'antre côté, l'endoderme se trouve immédiatement accolé à la face profonde de l'ectoderme dont il n'est séparé que par la membrane sans structure. Quand, par exception, l'organe testiculaire se développe de cette manière an lieu d'apparaitre à l'extrémité du sporosac, ce qui a licu dans l'immense majorité des cas, il peut être situé de manière à ne pouroir être aperçu sur une coupe optique du sporosac. Mais il est toujours possible, 


\section{(46)}

ch changeant la position du gonophore, de s'assurer de la présence de l'organe testiculaire. Je n'ai pas trouvé un scul sporosac dans lequel cet organe fit défaut.

Les lames testiculaire et médusoïde s'amincissent beaucoup dans le cours de l'évolution du sporosac, et quand le gonophore est arrivé à maturité, elles se trouvent réduites à l'état de pellicules très-fines qui se confondent en apparence et qui ont mime souvent un aspect fibrillaire, à raison de l'allongement considérable et de l'aplatissement des noyanx. Ceux-ci apparaissent alors à la coupe optique comme de petits bàtonnets réfríngents.

Jai cru plus avantageux d'exposer sans interruption le développement de l'organe testiculaire des sporosacs femelles, afin de rendre son histoire plus intelligible et de ne pas ètre obligé de scinder dans la suite l'exposé du déreloppenent de l'œuf.

Je reviens maintenant à la description de la figure 4, pl. I. Ce gonosome porte trois sporosacs inégalement développés. J'ai encore à faire connaitre la constitution du sporosac (c).

Ce gomophore, beancoup plus volumineux que celui que nous avons décrit en dernier lieu, affecte une forme globuleuse et sa base étranglée constituc pour l'organe une sorte de pédicule. Ce nouvel aspect du sporosac est principalement déterminé par les modifications qui se sont produites dans la constitntion de l'endoderme. L'ectoderme a subi aussi quelques changements; mais ils se résument dans un épaississement peu consillérable, dépendant $1^{\circ}$ de ce que toutes les eellules de lectoderine terminées extírieurement par une surface plane dans les sporosacs plus jeuncs, sont devemus convexes; $2^{\circ}$ de ce que des vacuoles remplies d'un liquide hỵalin ont apparu à leur intérieur. A l'extrémité des sporosacs se trouve un renflement de l'ec- 
toderme (fig. 16, 18, 19, 20), dans lequel il est impossible de distinguer aucune trace de territoires cellulaires, même en s'aidant de la solution d'acide osmique, si émineminent favorable pour faire apparaitre les contours des cellules de l'ectoderme. Ce renflement terminal parait formé par des cellules confondues en une masse protoplasinique commune dans laquelle on observe ça ct là quelques noyaux. Leur protoplasme est très-finement, mais uniformément granuleux; il ne renferme ni vacuole, ni liquide hyalin. Dans quelque cas j'ai observé dans ce renftement terminal une dépression médianc correspondant peut-être à l'entrée de la fente testiculaire.

Dans le pédicule, aussi bien qu’à la surface de la partic renflée du sporosac, on observe des fibrilles mnsculaires pàles et grêles. Disposées parallèlement les unes aux autres dans le pédicule, clles se distribuent en divergeant à la surface des glomérules, comme si elles étaient autant de méridiens tracés sur un globe.

Sous les fibrilles musculaires se voit la lamelle liyaline qui, au nivean du pédicule, présente à peu près l'épaisseur qu'elle affecte dans toute l'étendue de la région germinative, tandis qu'elle paraît plus inince dans le renflement terminal des sporosacs. Sous la membrane hyaline se voit la lame médusoïde, puis la lame testiculaire déjả peu distincte de la première, sauf cependant près de l'insertion des lames mélusoïdes sur l'endoderme. Celles-ci s'insèrent sur la membrane endodermique par une lase élargie (fig. $4, c$ et fig. 21). C'est toujours le cas dans les sporosacs bien déreloppés. Immédiatement en dedans on voit la lame testiculaire se terminer brusquement. Ces deux membranes sarrètent tonjours au sommet du pédicule, au point où celui-ci commence à s'élargir pour former la partic terminale renfléc du sporosac. Dans le pédicule l'endoderme est immé- 
diatement appliqqué contre la face interne de la inmelle lugaline; dans le renflement terminal du sporosac, il en est séparé par les lames médusö̈lde et testiculaire.

Dans le pédicule les caractères de l'endoderme sont restés à peu près les mèmes que dans les jeunes sporosacs. On y trouve des cellules semblables à de jeuncs wufs au début de leur développement; elles sont fusiformes et se trourent intercalées, dans l'épaisscur de l'endoderme, entre les cellules prismatiques grềles. Cependant celles-ci sont notablement plus larges et, par conséquent, proportionnellement moins longues que dans la réggion germinative; leur noyan est plus volumineus. Mais ce pui les distingue surtout des cellules de la régron cambiale, c’est ģı au contact de la cavité digestive, elles se sont chargées de globules réfringents et de granules pigmentaires. Par lá clles ressemblent déja beancoup aux cellules de l'endoderme lasilaire.

Au contraire, dans le sporosac proprement dit, les jeunes wuf's ont notablement grandi; leur corps primitivement peu déreloppé et peu granuleux s’est considérablement accru; il s'est chargé de granules vitellins au point d'avoir perdu en grande partic sa translucidité. Malgré cette circonstance on y distingue toujours les grandes vésicu'es germinatives; elles sont restées parfaitement sphériques, tont à fait transparentes et pourvucs d'un grand nuclécle à nucléolin (granule de Schrön). Quand on traite parl'acide acétique très-faible (sol.de 1 pour 10,000 ) le vitellus séclaircit et l'on reconnait alor's qu'il existe dans les vésicules germinatives, à côté du nucléole, plusieurs granules réfringents, notablement plus petits que le nueléole proprement dit, mais qui paraissent formés de la mème substance que lui. Ie n'ai pas pu un'assurer si ces globules existent dans les 
résicules germinatives avant l'addition de la solution acidulée. Toujours est-il qu'on ne voit pas de traces de ces nucléoles secondaires dans les vésicules plus jeunes, el que dans les aufs plus avancés ces corpuscules se tronvent plus volıminenx à côté du nucléole auquel ils finissent par ressembler complétement. J'ai ru aussi que le nucléolin augmente de volume dans le nucléole à mesure que l'œuf approclie de sa maturité. Dans des oufs complétement déreloppés, la substance réfringente des nucléoles est souvent réduite à une mince conche qui entoure comme une fine pellicule le granule de Schrön.

Les œufs prement peu à peu (fig. 18, 19, 21 et fig. 1 d et c) une forme arrondie. En augmentant de volume principalement dans leur partie moyenne, les orules ont exercé une pression croissante sur les cellules de forme prismatique qui les séparaient les uns des autres. Ils ont fini par étrangler ces cellules et par les diviser en deux parties: l'une interne, qui continue à circonscrire le diverticule de la eavité digestive; l'autre externe adjacente \&i la lame testiculaire. Les portions internes sont beancoup plus rolumineuses; la plus grande partie des cellules prismatiques primitives est refoulée vers l'intérienr. II est probable qu'un certain nombre de ces cellules protoplasmiques, grâce à la contractilité de leur substance, se retirent vers l'intérieur plutôt que de se laisser étrangler. Quoi qu'il en soit, les wufs semblent progressirement expulsés de l'endoderme ou plutôt cette membrane se reconstitue autour de la cavité digestive du sporosac de façon à recourrir complétement les œufs d'un côté. Dès lor's les œuf́s semblent avoir été refoulés entre l'endoderme régénéré et la lame testiculaire. En réalité ils restent plongés dans l'endoderme modifié : car les cellules à petits novaux qui per- 
sistent dans les espaces interovulaires situés sous la lame testiculaire, ne sont que les restes des cellules prismatiques primitives de l'endoderme (lig. $19 \mathrm{et} 20$, cio). Ces espaces interovulaires sont circonserits d'un côté par la lame testiculaire, de l'antre par les œuf́s mêmes qui, à raison de leur forme oroüde, ne peurent pas se toucher suivant toute leur surface. Mais comme es espaces sont extrêmement petits, qu'ils ont toujours passé inaperçus aussi longtemps que l'on n’a pas étudié suffisamment le déreloppenent des sporosacs, il a été affirmé que les œufs se trourent toujours chez les Ifydrö̈des entre l'endoderme et l'ectoderme. Les lames testiculaire et médusoïde, à raison de lenr peu d’épaiseur, ne se reconnaissent que si l'on a suivi leur dévelopjement. En fait, elhaque cuf' n'est en contact avec la lame testiculaire que par cette partic agrandie de sa surface qui s'appliquait con tre la lamelle hyaline, quand il était eneore simple cellule endodermique. Par tout le reste de lenr sur. face les wufs demeurent en contact arec des éléments endodermiques: les cellules interorulaires, les enfs roisins et les cellules de l'endoderme régénéré.

L'endoderme régénéré à l'intéricur du sporosac constitue ce que Allman a appelé le spadix du sporosac. II se moule sur les œufs et tantôt il s'élère jusqu’au sommet du gonophore (fig. 19), tantôt il en reste séparé par un œuf (fig. 20). Le spadix est formé par des cellules cylindroüles pourves clacune d'un petit noyau, de globules réfringents et de granula tions pigmentaires. Ces cellules sont aussi ponrvues d'un cil et elles ressemblent beaucoup aux cellules épithéliales de la portion hasilaire du corps. Cet épithélium se continue avec celui du pédicule qui, à son tour, passe à l'épithélinm de la région gastrique.

J'ai exposé jusqu'iei l'érolution des sporosacs aux diffé- 


\section{( 51 )}

rents moments de leur développement; leur étude comparative a suffi pour montrer:

$1^{\circ}$ Que les œufs sont primitivement de simples cellules endodermiques et qu'ils se trouvent déjà différenciés dans l'épaisseur' de l'endoderme avant que le sporosac ait commencé à se former;

20 Oue les oufs, en se développant, cessent de concourir à la délimitation de la cavité digestive. Il est probable qu'ici comme chez les Spongiaires, le cil de la cellule endodermique est successivement retiré comme le serait un pseudopode et qu'il finit par se fondre dans te corps protoplasmique de la cellule en voie de se transformer en œuf;

$5^{\circ}$ Que les ceufs semblent venir prendre position entre l'endoderme et l'ectoderme, tandis qu'en réalité ils r'estent entourés sauf là où ils sont en contact arec la lame testiculaire par des éléments de l'endoder'me;

4. Qu'une partic seulement des jeunes œuf's qui se trouvent primitivement dans le sporosac atteignent leur maturité. Les autres, logés dans l'endoderme du pédicule, restent stationnaires au lieu de se développer et bientôt ils s'atrophient; ils reprennent probablement les earactères des cellules épithéliales voisines; ce sont des œuls avortés;

כ̋ Entre l'ectoderme el l'endoderme se développe aux dépens de l'ectoderme un testicule rudimentaire, qui se réduit dans le sporosac adulte à une mince lamelle cellulaire. Il en est de mème de la lame médusoïde, dépendance de l'endoderme, qui va s'interposer entre la lame testiculaire et la membrane liyaline. Celle-ci conserve toujour's sa mème position à la face profonde des cellules de l'endoderme, dont elle n'est séparée que par la couche musculaire. C"est seulement quand tous les cufs sont dégì 
formés que l'organe testiculaire commence à se montrer. au sommet du sporosac. Il n’y a donc aucun lien génétique cutre cette dépendance de l'ectoderme et les produits sexuels femelles.

Pour terminer je dois dire encore quelques mots des parois du corps dans la région germinative, après la formation des premiers sporosacs. Cliez des individus comme ceux que j’ai figurés (pl. I fig. 2, 3 et 4), la raie zone germinative se trouve entre les lignes horizontales $x \mathrm{et}$ $y$, y passant par le sommet de l'entonnoir qui surmonte la cavité gastrique. En effet dans cette région l'endoderme est constitué par de jeunes aufs déjà caractérisés par leur helle vésicule germinative et par des cellules prismatiques allongées. C'est dans cette région seulement que se forment de nouveaux sporosacs d'après le procédé que j’ai longuement décrit. Jamais un jeune sporosac ne se trouve dans un autre point du corps du gonosome. Plus bas, au contraire, au-dessous de la ligne $x$ se voit dans la tig. 4 un sporosac bien développé; cliez des gonosomes adultes on en voit souvent un grand nombre (fig. 1); j'en ai compté jusqu’à douze, insérés au-dessous de celte ligne. Ils sc trourent fixés alor's en des poiuts du corps où l'endoderme présente tous les caractéres de l'épithélium que j’ai décrit comme caractérisant la région basilaire ou gastrique du gonosome. Il y a décroissance réggulière et progressive de has en haut quant au volume et au développement des sporosacs. En dessous des sporosacs les plus avancés on cn trouve souvent un ou plusieur's qui, ayant éracué leurs œufs, subissent une véritable dégénérescence. Ce sont des gousses llétries qui ont laissé échapper leur semence; je les appellerais volontiers des corps jaunes, si je ne craignais 


\section{( 55 )}

d'appliquer un mot ponrvu d'une signification anatomique bien précise à un organe qui a une tont autre valeur morphologique. Ces sporosacs létris sont physiologiquement a la région germinative de notre gonosome, ce que les corps jaunes sont à l'ovaire.

$\mathrm{Si}$, d'un autre còté, l'on considlère que la région gastrique. d'un gonosome est d'autant plus longue que celui-ci a porté un plus grand nombre de sporosacs, landis que la région cambiale conserve tonjours la même longucur, on reconnaitra que la région gastrique s'étend progressivement aux dépens de la région germinative au fur et à mesure que se forment les sporosacs; el qu'en même temps, la région germinative envahit progressivement la région cambiale. Celle-ci se déplace peu à peu de bas en haut à la suite de la transformation progressive de nonvelles cellules de lendoderme cambial en orules.

L'endoderme de la région cambiale se régénère luimime, probablement par multiplication cellulaire, absolument comme le font les cellules du cambium des dicotylédones qui donnent naissance ì de nourelles couches d'écorce et de bois, tout en conservant la mêne épaisseur et la même puissance génératrice. C’est cette comparaison physsiolngique avec le cambium végétal qui m’a déterminé à donner à cetle région du corps le nom de région cambiale.

II ne se déreloppe pas de sporosacs sur tont le périmètre de la région germinative, quoique sur tout son pourtour l'endoderme renferme de jeunes œufs. Or, les jeunes œuf's ne múrissent que dans les sporosacs; il faut done qu'une partie des jeunes cufs arortent pendant la transformation progressive de lépithélium germinatif en épithélimm gastrigue. On reconnait en effet, quand on examine la région germinative, que les plus grosses vésicules germinatives 


\section{$(5 t)$}

se trouvent dans la partie movenne de cette région. Vers la ligne $x$ elles sont moins volumineuses et passent insensiblement aux noyaux des cellules gastriques. Au contraire, les noyaux de transition qu'on trouve vers la ligne $y$ sont des noyanx de cellules cambiales en roie de derenir des résicules germinatives. L.es ovules avortés deriennent-ils de simples cellules gastriques ou bien s'atrophient-ils connplétement de façon à disparaître? Je ne pourrais encore pour le moment donner à cette question une réponse catégorique.

Le fail de l'avortement physiologique d'un grand nombre d'œufs en roie de développement est fréquent chez des formes appartenant à diver's types d'or'ganisation. Combien de milliers de vésicules de de Graaf avortent daus l'ovaire des mammifères! Combien on en troure encore, tout au début de leur développement chez la femme arrivée à l'àge critique, ou cliez la poule perdant les attributs extérieurs de son sexe! Les prétendues cellules vitellogènes des insectes ne sont-elles pas des ceuf's avortés? II en est certainement ainsi des trois cellules qui, chez les Daphnies, sont constamment accolées à l'œeuf en roie de développement (P.-E. Müller); chez un certain nombre de Phyllopodes et spécialement chez les Apus le nıème fait a été établi par les belles observations de ron Siebold.

Je puis terminer ici l'étude du gonosome femelle et des sporosacs auxquels il donne naissance. Car le gousome porteur d'un grand nombre de sporosacs ne diflère guère de celıi que j’ai représenté (pl. I, fig. 4), qu'en ce que chez. lui la région gastrique est nolablement plus longue et en ce que dans la partic de cette région qui avoisine la région germinative s'insèrent un grand nombre de sporosacs l’antant plus volumineu. quiils se trourent insérés plus bas. 


\section{( รัว )}

Je ponrrais aussi compléter ici la description de l'ouf et exposer les plrénomènes de sa maturation progressire; mais je ne reux moccuper dans ce travail que de la question de l'origine des produits sexuels. Je signalerai senlement, en terminant, ce fait important qu'il n'existe autour des cufs arrivés à maturité ni mombrane, ni rien qui rappelle l'albumen des oiseanx. Quelques antenr's ont décrit une membrane mince autour de l'œuf' de certains Coclentérés. Je ne sais si cette membrane existe quelquefois; mais elle manque positivement chez les Iydractinies. Huxley a démontré qu’elle fait défant chez les Siphonophores; Gegenbauer pense qu'elle n'cxiste ni cliez les Calycoplıorides ni clıez les Physsophorides; Allman et Schulze l'ont cherchée en rain chez les Cordylophores.

Eulin, Heckel a établi que l'absence de toute membrane vitelline et de tout chorion autonr de l'weuf des Spongiaires est une particularité distinctive de l'ouf de ces Zoopliytes inférieurs.

\section{Gonosome mile.}

Les colonies mâles présentent comme les colonies femelles, le phénomène du polymorphisme. Toute colonic mâle se compose d'Hydrantlies, de gonosomes et de zooïdes spiralö̈des. On y trouve des gonosomes à tous les états de développement et il n'est pas difficile d'en décourrir chęz lesquels on naperçoit encore ancune trace de sporosac. Le corps de ces jeunes individus est tout à fait constitué comme celui des jeunes gonosomes de sexe femelle; indépentamment de l'extrémité supérieure renflée el qui affecte plus ou moins la forme d'un chou-fleur, on peut distinguer une région cambiale, une région germinative ot une région gastrique. La cavitégastrique est assez large; 


\section{( 56 )}

elle se rétrécit brusquement à son extrémité supéricure el se termine par un entonnoir renversé dont le sommet correspond à la limite de la région cambiale. Quant à la structure des gonosomes mâles, elle est la mème que celle des femelles : l'ectoderme présente chez cux les mêmes caractires; les pseudotentaculesne sont que des épaississements de cette couche cellulaire dans lesquels se déreloppe une grande quantité d'organes urticants. Sous l'épiderme se trouve la couche musculaire appliquée à la face externe de la membrane hyaline. L'endoderme est constitué chez le mâle comme chez la femelle, a u moins en ce qui concerne les régions gastrique et cambiale. Par contre, daus cette partie du corps du gonosome qui correspond à la rígion germinative de la femelle, on ne trouve pas d'orules sembles à ceux que j’ai décrits plus haut. Les cellules qui constituent l'endoderme de cette région ressemblent en tous points à l'épithélium endodermique de la région cambiale. Néanmoins on y rencontre ça et là des cellules qui ont des noyaux notablement plus grands à côté d'autres qui, à ce point de rue, ne diffèrent en rien des cellules cambiales ordinaires ( pl. II, fig. 4). Il est impossible de confondre arec de jeunes oufs les cellules endodermiques ì grands noyaux que l'on troure chez le mâle. Mais le fait que certaines cellules tendentà se différencier des cellules roisines, démontre éridemment que cette région est homologue de la région germinative du gonosome fomelle el que non-seulement la couche cellulaire aux dépens de la(juelle se forment les ovules chez les femclles, mais les ovules cux-mêmes se trourent chez le mâle plus ou moins différenciés. Je rappellerai, à ce sujet, une observation intéressante faite il $y$ a longtcmps par ron Wittich : il a démontré que, chez le Crapaud, le testiculc est enreloppé 
d'une couche cellulaire qui est homologue de l'ovaire de la fenelle; que dans cette conche apparaissent de véritables œufs qui penvent atteindre dans certaius cas un assez grand développement, de facon à ressembler complétement ì de jeunes œufs de la femelle, sans cependant atteindre jamais leur maturité chez le màle. Waldeyer a reconnu cette mème conche cellulaire représentant un ovaire rudimentaire clıez l'embryon mâle du poulet, voire même chez les mammifères. Bien plus, il a vin des éléments semblables ì de jeunes ocufs apparaitre chez le mâle et se former aux dépens de cette couche épithéliale superficielle. Je n’ai jamais trouvé chez le gonosome màle des Hydractinies de rais ovules semblables à ceux que j’ai décrits dans la couche germinative du gonosome femelle; mais les cellules à noyaux plus volumineux qui y existent constamment rejrésentent incontestablement l'une des phases de la transtormation des cellules endodermiques ordinaires en ovules. Si cette interprétation est exacte, l'on peut affirmer l'existence d'un ovaire rudimentaire chez le màle des IIydrö̀des. Ce qui confirme cette manière de voir, e'est que dans le déreloppement des sporosaes mâles, l'endoderme germinatif se comporte exactement comme daus les jeunes sporosacs femelles. Lne autre raison qui doit faire admettre chez le unàle une région germinative homologue de celle du gonosume femelle, c'est que les sporosacs mâles ne se développent jamais que dans une zone étroite, parfaitement linitée, qui correspond exactement à la zone germinative de la femelle.

Le mode de développenent des sporosacs est identique à celui que j'ai décrit chez le gonosome femelle. Ces sporosacs sont, au début, de simples diverticules des parois du corps, formés à la fois par l'ectoderme et par 


\section{(}

l'endoderme; la cavité digestive s’y prolonge et s’y termine en un cul-de-sae circonscrit par l'endoderme soulevé (pl. Il, fig. 1 a el fig. 5). Le sporosae passe sueci'ssivement dans le eours de son évolution par la forme d'un lubercule eonoïde, d'un ovö̈le à grand axe plus ou moins perpendieulaire à l'axe du corps du gonosome; enfin il derient globulaire en mème temps quil se pédiculise. Tant que le sporosac a la forme d'une oroïle allongé, te prolongement de la eavité digestive qui s'étend à son intírieur se réduit à une fente, l'endoderme étant alor's adossé à lui-mème, de façon à effacer plus ou moins complétement la eavité. Quand, au eontraire, l’ovoïde se rétrécit à sa base pour devenir un véritable pédieule el quil se renfle dans la plus grande partie de son étendue pour eonstituer le sporosac proprement dit, la cavité digestive du gonophore se dilate progressirement.

Dans inn jeune sporosac semblable à eelui que jai dessiné (pl. II, fig. 7,8 et 9), il s’est formé aux dépens des cellules de l'ectodernie et de la même manière que. je l'ai déerit plus haut pour le sporosae femelle, un bourgeon eellulaire arrondi qui déprime l'endoderme. Ce bourgeon est, dès le début, un peu plus volunineux que chez la femelle; il est constitué par quelques eellules ectodermiques de forme eonoïde dans lesquelles on distingue deux el quelqnefois. trois noyaux. J'ai rn aussi, dans quelques cas, ce tubereule résulter d'une réritahle invagination de l'eeloderme (fig. 8). Ce bourgeon, qui n'est autre ehose que le testicule en roie de développement, s'accroit par multiplication eellulaire; il sélargit et se développe dans tous les sens. L'endoderme se moule réritablement sur lui de façon à former exactement te contre-moule du hourgeon. De lia résulte la production de 


\section{( 59 )}

deux cornes endodermiques, les lames méd usoïdes, (pl. II, fig. 9, 10 et suivantes ).

Le bourgeon testiculaire s’aplatil et s’étale; il se glisse véritablement entre l'endoderme et les deux cornes qui en partent latéralement. La face profonde du bourgeon, d'ahord convexe, devient concave, et tandis que prinitive" ment l'endoderme avait l'air de se mouler sur le hourgeon, c'est maintenant le bourgeon qui paraît se mouler sur la partie terminale de l'endoderme (pl. II, fig. 1J).

Le bourgeon prend ainsi la forme d'une calotte ou d'une soucoupe qui, à la section optique, a l'apparence d'un croissant. Les cornes endodermiques s’élèvent rapidement, de façon à étrangler de plus en plus le pédicule par lequel l'organe testiculaire se trouve encore en continuité arec le tissu ectodermique. Ce pédicule se rétrécit et l'organe testiculaire finit par se détacher complétement de l'ectoderme. Les deux cornes ou plutôt les lanıes médusoïdes se sont alors soudées entre elles et le testicule isolé se trouve entouré de toutes parts par des éléments endodermiques. Mais les lames médusoïles, formées par une seule rangée de cellules plates, sont très-minces et par là le testicule parait situé entre l'endoderme et l'ectoderme.

A ce moment, le testicule est formé d'un grand nombre de cellules dont les noyaux seuls sont hien distincts (pl. Il, fig. 10 et suir.). Ces noyaux sont extrêmement rapprochés l'un de l'autre. Ils ont une forme sphérique, des contours pầles et ils sont encore pourvus d'un petit nucléole (pl. II, fig. 10, 11 et 12). Ils paraissent logés dans une substance protoplasmique commune assez réfringente. Je n’ai janais réussi, ni en employant l'acide osmicque, ni en me serrant de l'acide acétique faible, ni en traitant par 


\section{(60)}

les matières colorantes, à distinguer les circonscriptions cellulaires des éléments du lesticule. Je ne sais si les cellules sont distinctes ou si elles sont différenciées seulement par leur's noyaux.

L'organe testiculaire grandit; il devient une conclie cellulaire épaisse, étalée comme un gros bonrrelet tout antour de l'extrémité du spadix endodermique ( $\mathrm{pl}$. JI, figg. 12, 15 et 14 et fig. 1 e); en même temps, les lames médısoïdes s'amincissent notablement. Dans les jeunes sporosacs, quand le testicule est cncore rudimentaire, il y a continuité entre le tissu cellulaire des cornes de l'endoderme et la conche épithéliale du spadix; mais quand le testicule a atteint le déreloppement de ceux que j’ai représentés (figg. 12, 15 et 14), les cornes sont nellement séparées du spadix sur les parois'duquel elles s'insèrent par une lase ćlargie.

L'ectoderme des sporosacs mâles diffère assez notablement de celui des femelles. Dans les jeunes sporosacs màles, il est proportionnellement plus épais, tandis que dans les sporosacs plus arancés il est notablement plus mince. Le déreloppement de l'ectoderme au point de vue de son épaisseur anx différents moments de l'évolution des sporosacs est donc différent dans les deux sexes. II semble qu'il $y$ ait un rapport inverse de développement entre l'organe testiculaire et l'ectoderme. Quand le testicule est rudimentaire, l'ectoderme est plus épais; c’est le contraire quand le testicule sc développe. Je n’ai jamais trouvé autour d’un sporosac mâle hicn développé un ectoderme formé de ces grandes cellules conveses et pourrues d'un système de vacnoles remplies d'un liquide lịalin. Ces cellules sont toujours plates, très-larges, peu nombrenses et nettement circonscrites (pl. I, fig. 5). F. E. Schulze a 


\section{(61)}

signalé le mème fait dans les gonophores mâles du Cordylophora.

Quant aux cellules de l'endoderme, elles se modifient principalement en ce que leur largeur augmente, en ce qu'elles se creusent de vacuoles et en ce qu'elles se chargent, au contact de la cavité digestive, de globules réfringents et de granules pigmentaires d'une couleur rose. C'est celte matière pigmentaire répandue dans tous les zooïles des colonies màles et femelles, principalement dans les cellules endodermiques de la région gastrique, qui donne à ces polypes leur teinte rosée, couleur de chair. Qunand on examine un sporosac mâle à un faible grossissement, on distingue toujours le spadix du sporosac, tranchant par sa coloration rose, sur le blanc mat de la calotte testiculaire.

Déjà dans un jeune testicule comme celui qui se trour. liguré (pl. II, lig. 11), on distingue une petite fente semblable à celle qui existe toujours dans l'organe testiculaire du sporosac femelle. Mais dans l'organe testiculaire du mâle, la fente se troure toujour's près de la surface. Elle sépare de la plus grande partic de la masse cellulaire dn testicule une couche superficielle formée d'une seule rangéc de cellules. Cette fente se développe en même temps que le testicule et les cellules superficiclles forment, au tissu séminal, un véritable épithélium. Cet épithélium est homologue de l'ectoderme du sous-ombrelle des lléduses; le testicule lui-même peut être considéré comme représentant la couche ectodermique du manubrium d'une Néduse mâle; la fente testiculaire esı homologue de la qui, dans la Méduse, existe entre le manubrium el la face interne du manteau. Plyylogéniquement il faut con- 


\section{( 62 )}

sidérer le sporosac comme ayant précédé la Méduse. Le sporosac u'est que l'organe reproducteur qui a pu s'isoler et atteindre la forme de Méduse, grâce au mode de développement de l'organe testiculaire par invagination de l'ectoderme. La fente testiculaire n'est que le reste de la cavité résultant de cette invagination primordiale (Einstïl. pungshöhle). Le système des canaux radiés de la Méduse et le canal circulaire du bord de l'ombrelle se sont formés aux dépens de la lane endodermique que j'ai appelée pour ee motif la lame médusoïde. II en est de mème des cellules endodermiques qui constituent toujours la charpente des icntacules.

L'épithélium superficiel du testicule ne donne jamais naissance à des spermatozoïdes. Il subit, au contraire, dès que l'organe approche de sa maturité une sorte de dégénérescence graisseuse. Il n'est plus possible alors de distinguer les noyanx des cellules et l'on trouve dans ceue couche un grand nombre de globules très-réfringents de dimensions variables (pl. II, fig. 14).

Mes observations sur le développement des spermatozoïdes ne sont pas encore assez complètes pour me permettre d'en donner la description. Je signalerai seulement ce fait que les noyaux de la masse testiculaire deviennent tellement nombreux qu'ils finissent par se toucher, quant le testicule a atteint le développement que j’ai représenté (pl. II, fig. 14). Ces noyaux sont alors fort petits et ils paraissent dépourvus de nucléole. Les caractères des spermatozoüles sont semblables à ceux du Cordylophora et de l'Hydre d'eau donce; mais la tète me parait notablement plus petite chez. les Ilydractinies.

Habituellement le sporosac màle, arrivé à son complet développement, a une forme parfaitement symétrique : le 


\section{( 65$)$}

spadix occupe l'axe du sporosac et il est recouvert de toutes parts par le testicule qui se comporte vis-à-vis de lui comme un dé à coudre vis-à-vis de l'extrémité du dloigt qui le porte. Mais on trouve çà et là des sporosacs tout á fait dissymétriques $(1)$ l. 11 , fig. $1, d$ ); on reconnaît alors que le testicnle entoure à la manière d'un bourrelet dont le plan serait horizontal le spadix dont l'axe est supposé vertical. Dans ce cas le spadix est accolé contre l'ectoderme, et dans certaines positions du sporosac on roit le spadix, an milieu, se porter jusqu’à l'extrémité du sporosac; à droite el à gauche du spadix on distingue la coupe optique du bourrelet testiculaire. Quelquefois le testicule se trouve tout entier léveloppé sur l'un des côtés du sporosac et le spadix occupe l'autre côté. Ces différences dépendent exclusivement du mode de croissance et de la position du bourgeon testiculaire, an moment de son apparition. Elles sont du reste sans importance au point de vue morhologique.

\section{CONCLUSIONS.}

\section{Cliez les Hydractinies}

1. Les oufs se développent exclusivement aux dópens des cellules épithéliales de l'endoderme. Its restent, jusqu'au moment de leur maturité, entourés par les éléments le l'endoderme.

$2 \circ$ Le testicule et les spermatozoïdes se développent aux dépens de l'ectoderme; cet organe résulte de la transformation progressive d'un repli cellulaire primitivement formé par invagination.

$5^{\circ}$ Il existe dans les sporosacs femelles un rudiment d'organe testiculaire; dans les sporosacs mâles un ruli- 


\section{(64)}

ment d'oraire. Les sporosacs sont done morploologiquement hermaphrodites.

L'endoderme el l'ectoderme ont, au point de vue sexuel, une signification opposée. S'il est rai que les organes se forment par différenciation anatomique à la suite d'une division du travail physiologique, il faut admettre que, primitivement, l'ectoderme tout enticr était chargé de la fonction sexuclle màle, et qque l'endoderme remplissait la fonction sexuelle femelle. Si l'ectoderme peut être apjelé feuillet animal (nerveux et musculaire), parce que les cellules de l'épiderine, du système nerveux et du système musculaire résultent d'une différenciation progressive des cellules de l'ectoderme, ce feuillet doit être consiltéré en même temps comme le feuillet mâle L'endoderme est le feuillet femelle en même temps que le fenillet végétatif.

La fécondation consiste dans l'union d'un veuf', produit de l'endoderme arec un certain nombre de spermatozoïdes, produits de l'ectoderme; eet acte n'a d'autre but que de rassembler des éléments chimiques de polarité opjosée, qui, après a voir été réunis un instant dans l'œuf, se séparent de nouveau; car chez la plupart des animaux, dis que la division du vitellus en deux apparaitl, les éléments aux dépens desquels va se former l'ectoderme sont déji séparés de ceux qui vont fournir te feuillet interne de l'embryon.

L'individualité nouvelle se tronve réalisée au moment où l'union entre les éléments de polarité opposée s'opère, absolument comme la molécule d'eau se forme par l'union des atomes d'hydrogìne et d'oxygène.

Observalion. - Il résulte de toutes les dernières olsservations embryogéniques faites chez les vertébrés, que le feuillet moyen de ron Bä̈r et de Remali ne donne nais- 


\section{(65)}

sance qu’ả l'épithélium de la cavité péritonéale primitive. Or, d'après les observations de Waldejer, l'épithélium superficiel de l'ovaire des vertébrés n’est que cette partie de l'épithélium péritonéal qui recourre la plaque moyenne (Nittelplatte de Remali). Les observations de Götte, de Peremeschlio, de Schenk, d'OEllacher, de Rieneck, ont démontré que le feuillet interne et le feuillet moyen de Remak ne sont que des parties différenciées d'une même couclie cellulaire (l'endoderme) qui dérive tout entière du vitellus blanc chez les Batraciens. L'épithélium sexuel femelle, qui persiste à la surface de l'ovaire des mammifères, aux dépens duquel se forment les tubes ovariens, les résicules de de Graaf et les canaux de Iü̈ller, dérive done, en dernière analyse, de l'endoderme. Le testicule se forme aux dépens du canal de Wolff, d'après les obserrations de Waldeyer et de plusieurs autres embryogénistes. Or, MM. His, Hensen et Waldeyer font dériver le canal de Wolff du feuillet externe (ectoderne) par l'intermédiaire du cordon axial. Lee testicule dériverait donc aussi chez les vertébrés du feuillet ectodermique. Nes conclusions se trouveraient ainsi confirmées dès aujourd'lui en ce qui concerne l'embranchement des rertébrés. Les vertébrés sont les seuls chez lesquels, indépendamment des Zoophytes, l'origine des organes sexuels ait été recherchée; mais il est probable que ces résultats se vérifieront pour l'ensemble du règne animal. 


\title{
(66)
}

\section{EXPLICATION DES PLANCIIES.}

\author{
Plancue I.
}

\section{Exolectris echixar.}

Gonosomes femelles.

liin. 1. - Gonosome femelle adulte portant un grand nombre de sporosacs a différents états de développement. Les sporosaes sont d'antant plus développés qu'ils se trouvent plus éloignès de la région germinative situce entre les lignes $x$ et $y$. $a$, sporosae ell voie de développement et n'étant eneore qu'un simple divertieule de la ı'égion germinative (obj. ว̀ de IIartnach).

His. 2. - Jeune gonosome avant la lormation des sporosaes. - $a$, rigion gastrique. $-b$, région germinative limitée par les lignes $x$ et $y .-c$, région eambiale limitée par les lignes $y$ et $z$. d, renflement eéphalique en ehou-lleur, portant des pseudotentaeules (obj. 5 dle Hartnaek).

Fig. כ. - Jeune gonosome portant un sporosae en voic de léveloppement (b) (obj. 5 de Hartmaek).

Fig. f. - Gonosome plus avaneé portant trois sporosaes inegalement déreloppés $a, b, c$. Le sporosac $b$ montre l'organe testiculair't sous for'me d'un eroissant (obj. 5 de Hartnack).

liig. ว̈. - Cellules de l'eetoderme; $a$, rues de profil et reposant sur la lame lıyaline dont elles sont séparées par des libres museulaires; $b$, vues de faee (obj. $10 \mathrm{imm}$. Hartnaek; prèparation al l'aeide osmique).

Fig. 6. - Cellules de l'eetoderme à vaeuoles et gonflèes par un liquide Iryalin. - $a$, rues de profil; $b$, vues de face (meme grossissement et mème mode de préparation que précèdemment).

lïg. 7. - Cellules de l'endoderme dans la région gastrique vues de profil.

lig. 8. - Cellules de l'endoderme dans la region germinative. On distingue les ovules et les eellules prismatiques allongees.

liig. 9. - Mèmes cellules de la région eambiale. 


\section{$(67)$}

lïg. 10. - Mèmes cellulcs de la région eéphalique.

Les ligures 7 à 10 ont èté dessinées d'apris dles prẹparations de l'endoderme traité par une solution tris-l'aible $\frac{1}{10000}$ d'acile aectique et rues au moycu del'oljeetil a immersion 10 de llartnaek.

lig. 11. - Sporosac qui n’est encore qu'un simple diverticule du lube digestif (obj. 9. dc Hartuack).

Fig. 12 et 15. - Jeunes sporosaes. Les ovules s'eloignent de la caviti: digestive. L'organc testiculaire apparail comme simple epaississcincnt de l'ectodcrme. La figul' 15 montre cct organe sc formant par invagination.

lig. 14. - Phase ultéricurc du développenent dn sporosac. On voit dans le tubercule ectodermique la fente testieulaire. Lil ejpitlılium endodermique rccourre dejja la lace interne des $\mu^{\prime} u$ ts.

Figr. I\%̈. - Formation de l'organe testieulair" par invagination. La lente testiculaire s'ouve à l'extéricur du sjorosac.

Fig. 16. - L'organc testiculaire n'est pas sẻparé de l'eetoderme par des lames médusoïdes.

Fig. 17. - L'organc testiculaire détaché de l'ectoderme montre la fente testieulaire.

Fis. 18, 19 et 20. - Les plıascs ultéricures du développencut dés sporosacs; $c, i, o$, sout des cellulcs endodermiques intcrovulaircs.

Les figurcs 12 a 20 ont étè dcssinèes au même grossissement. (oljj. \& de Hartnack).

Fir. 2.1. - Portion d'un sperosac pour montr cr la lanelle liyaline $l, h, 0$, sous-ectodcrmique séparée lcs oeul's par la lame médusoïde Imel par l'organe testiculaire $0, l,(0) j .10$ a imm de llartnacli).

\section{PLAXCHE II.}

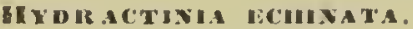

\section{Gonosomes mîles.}

Fig. 1. - Gonosome adultc portant plusicurs sporosacs majles a dillerents degrès de développentent en dessous dc la région germinative limitẻe par les lignes $x$ ct $y$. Les sporosacs sont lésignes par les letres $a, b, c, d, e$. Dans le sporosae $b$ on voit le testieule cn roic de développement aux déperss de l'velorlcrme (olıj. ö de Hartnaek). 


\section{( 68 )}

liz. 2. - Jeune gonosone mâle avant l'apparition des sporosaes. $a$, region gastrique; $b$, région germinative; $c$, region canbiale; $d$, renllement cephalique portant des pseudotentacules (ol)j. 5ै de Hartuaeh).

Fig. 5. - leune gonosone portant un sporosae oroïde et un autre apparaissant sous lorme d'un tubercule sur les parois de la régiou germinative (obj. ǰ de Hartnacli).

lïg. 4. - Cellules endodermicyues de la région cambiale folj. 10 à inın. de Ilartnack). Certaines cellules renferment des noyaux plus volumineux (ovules).

Fiz. §. - Fibres musculaires montrant leur contimuite avec des cellules de l'ectoderme. Eilles provienment de la région gastrique d’un \%oö̈de sjiraloüde. Préparation obtenue par dilaeération apres maciration pendant deux jours dans une solution de ehlorure de platine at dacide ehromique (obj. 10 it imn. (le Hartuack).

Fig. 6. - Membrane hyaline montrant la striation longitudiuale de sa faec externe. Memes rensejgnements que pour la figure $\mathfrak{i}$.

Fis. 7,8 et 9. - L'organe testieulaire a son début. Il se forme aux dépens (le l'ectoderme soit par épaississement (fig. 7 et 9), soit par invagination (fig. 8).

Fig. 10. - Le testieule gr'andit et s'aplatit.

Figr. 11. - Le testieule prend la lorme d'une ealotte. Il se pédiculise et l'on distingue nettement les lames medusoïdes. La fente testiculairc est très-apparente.

Fig. 12, 15 et 14. - Déreloppement ultérieur des sporosaes mâles.

Les figures 7 à 14 sont dessinèes au mime grossissement (olj. 8 de Hartuaeh). 



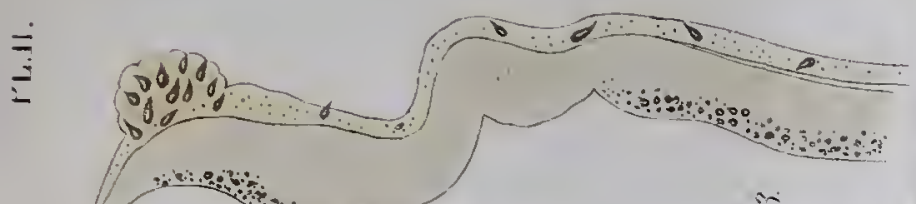
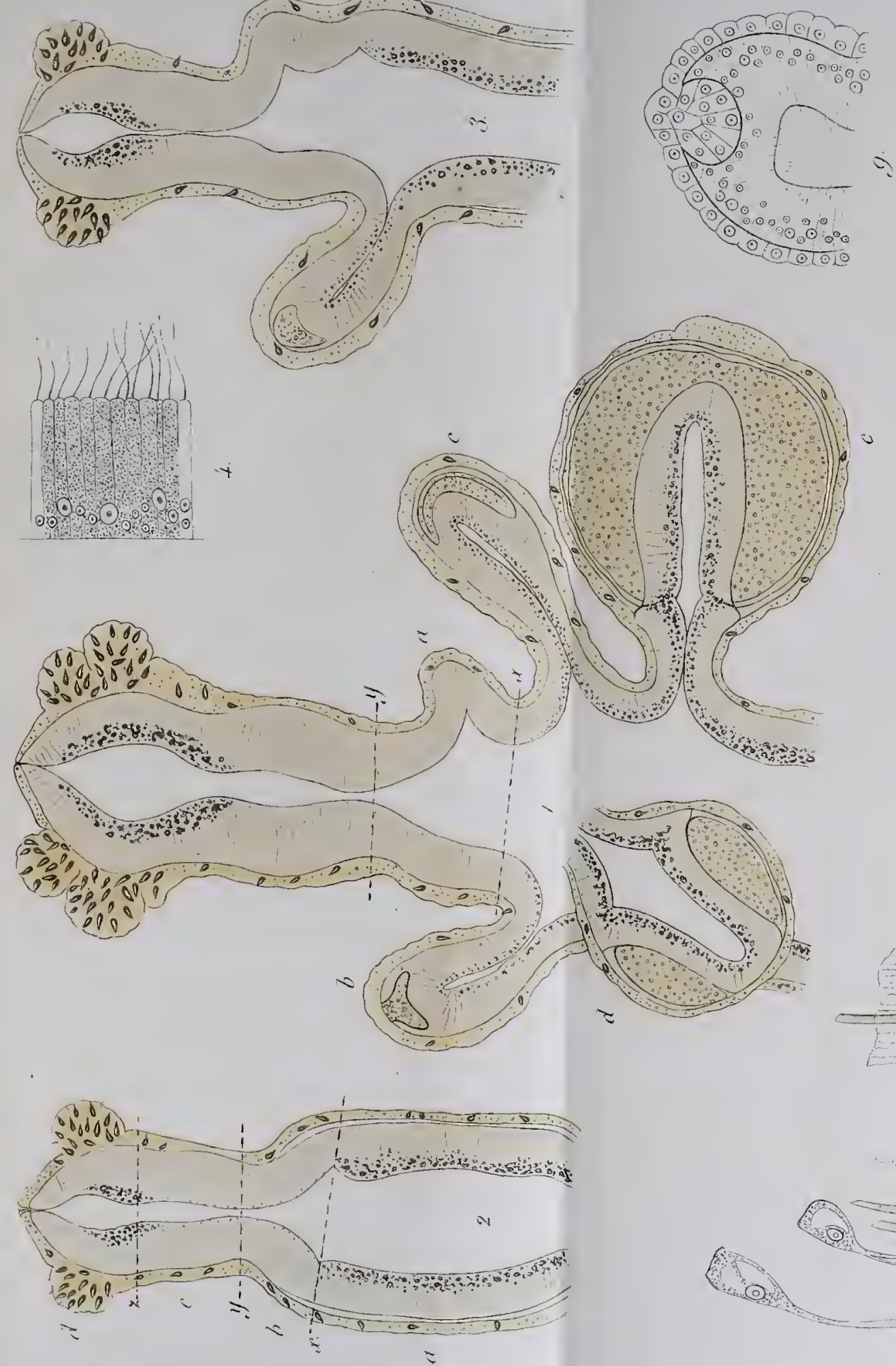
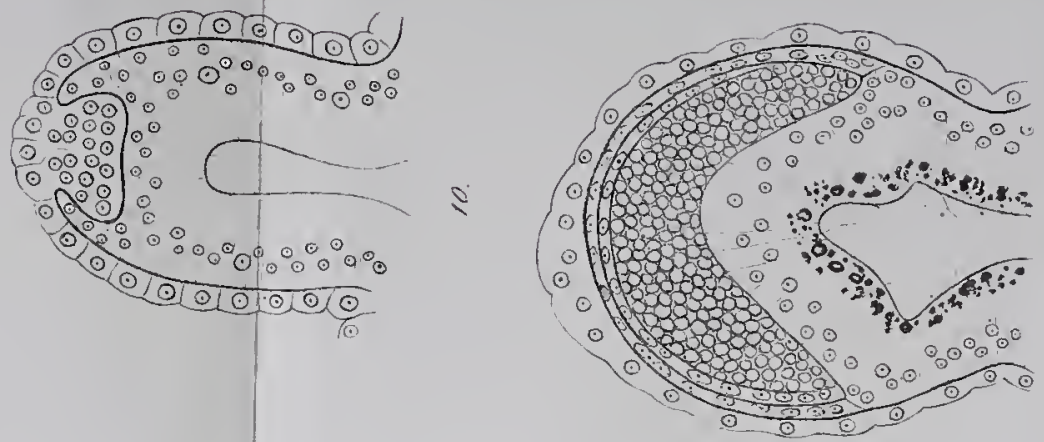

इै
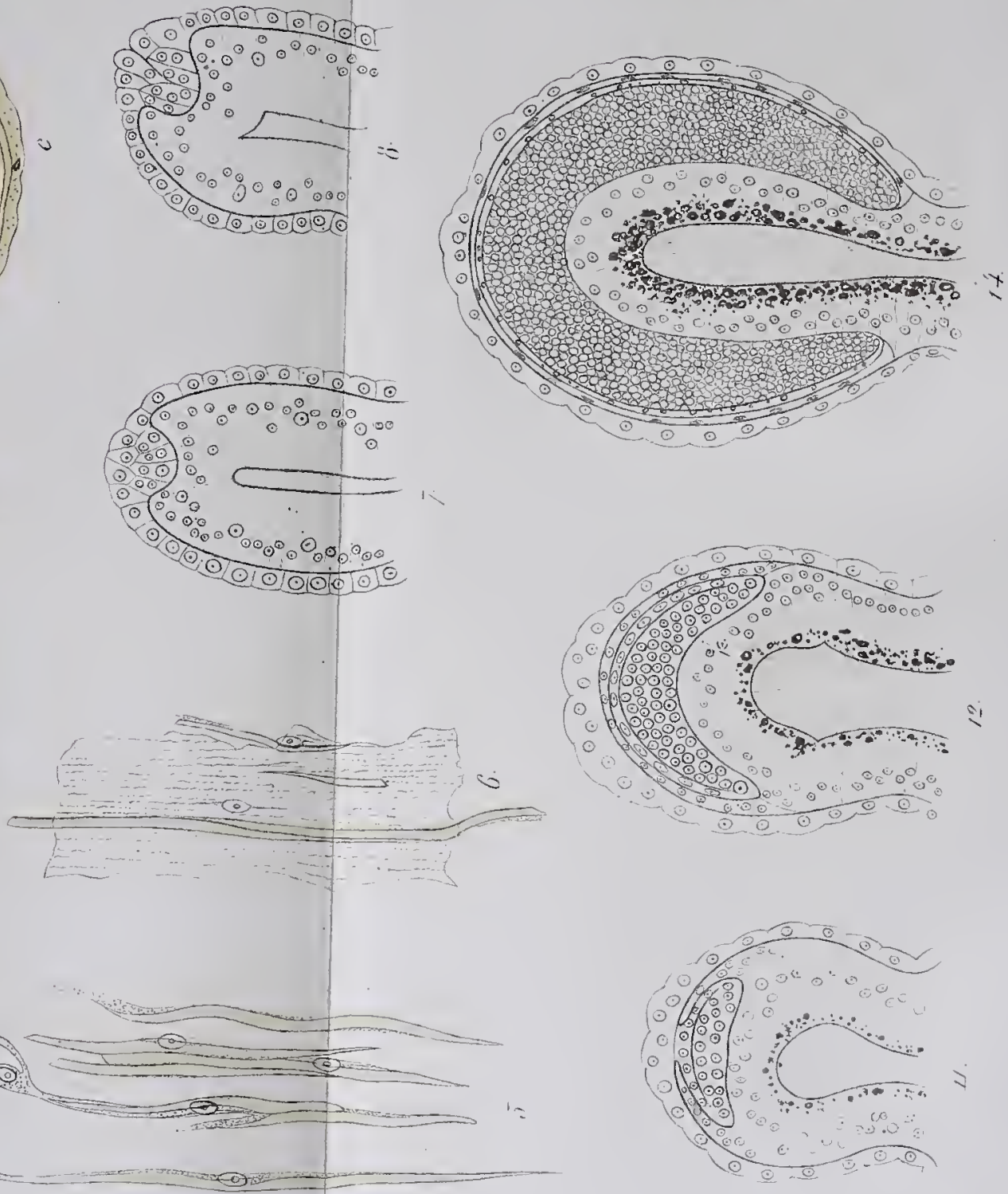
